\title{
Relic Neutrino Absorption Spectroscopy
}

\author{
Birgit Eberle and Andreas Ringwald \\ Deutsches Elektronen-Synchrotron DESY, Notkestr. 85, D-22607 Hamburg, Germany \\ Liguo Song \\ Department of Physics \& Astronomy, Vanderbilt University, Nashville, TN 37235, USA \\ Thomas J. Weiler \\ Department of Physics \& Astronomy, Vanderbilt University, Nashville, TN 37235, USA and \\ Kavli Institute for Particle Astrophysics and Cosmology, \\ Stanford Linear Accelerator Center, 2575 Sand Hill Road, Menlo Park, CA 94025, USA
}

\begin{abstract}
Resonant annihilation of extremely high-energy cosmic neutrinos on big-bang relic anti-neutrinos (and vice versa) into Z-bosons leads to sizable absorption dips in the neutrino flux to be observed at Earth. The high-energy edges of these dips are fixed, via the resonance energies, by the neutrino masses alone. Their depths are determined by the cosmic neutrino background density, by the cosmological parameters determining the expansion rate of the universe, and by the large redshift history of the cosmic neutrino sources. We investigate the possibility of determining the existence of the cosmic neutrino background within the next decade from a measurement of these absorption dips in the neutrino flux. As a by-product, we study the prospects to infer the absolute neutrino mass scale. We find that, with the presently planned neutrino detectors (ANITA, Auger, EUSO, OWL, RICE, and SalSA) operating in the relevant energy regime above $10^{21} \mathrm{eV}$, relic neutrino absorption spectroscopy becomes a realistic possibility. It requires, however, the existence of extremely powerful neutrino sources, which should be opaque to nucleons and high-energy photons to evade present constraints. Furthermore, the neutrino mass spectrum must be quasi-degenerate to optimize the dip, which implies $m_{\nu} \gtrsim 0.1 \mathrm{eV}$ for the lightest neutrino. With a second generation of neutrino detectors, these demanding requirements can be relaxed considerably.

PACS numbers: 14.60.Pq, 98.70.Sa, 95.85.Ry, 95.35.+d
\end{abstract}

\section{INTRODUCTION}

Neutrinos are the elementary particles with the weakest known interactions. Correspondingly, they can propagate to us through the cosmic microwave and neutrino background ( $\mathrm{CMB}$ and $\mathrm{C} \nu \mathrm{B}$, respectively) without significant energy loss even from cosmological distances. A possible exception to this transparency is resonant annihilation of extremely high energy cosmic neutrinos (EHEC $\nu$ 's) on big-bang relic anti-neutrinos (and vice versa) into Z-bosons [1-4]. This occurs near the respective resonance energies,

$$
E_{\nu_{i}}^{\mathrm{res}}=\frac{m_{Z}^{2}}{2 m_{\nu_{i}}}=4.2 \times 10^{22} \mathrm{eV}\left(\frac{0.1 \mathrm{eV}}{m_{\nu_{i}}}\right),
$$

with $m_{Z}=91.2 \mathrm{GeV}$ denoting the mass of the Z-boson [5] and $m_{\nu_{i}}(i=1,2,3)$ the non-zero neutrino masses for which there is rather convincing evidence inferred from the apparent observation of neutrino oscillations [5]. On resonance, the corresponding cross-sections are enhanced by several orders of magnitude. This leads to a few percent probability of annihilation within the Hubble radius of the universe, even if one neglects further enhancing effects due to cosmic evolution. Indeed, it appears that - apart from the indirect evidence to be gained from cosmology, e.g., big-bang nucleosynthesis and large-scale structure formation - this annihilation mechanism is the unique process [82] having sensitivity to the $\mathrm{C} \nu \mathrm{B}$ [1]. Moreover, observation of the absorption dips would present one of the few opportunities to determine absolute neutrino masses [9]. However, the mechanism requires that there exists a sufficiently large $\mathrm{EHEC} \nu$ flux at the resonant energies of Eq. (1). One of the purposes of this work is to quantify how large this $\mathrm{EHEC} \nu$ flux must be.

Since the original proposal in 1982 [1], significant advances have been made in theoretical and observational cosmology, experimental neutrino physics, and EHEC $\nu$ physics. Each of these three areas impacts immediately on the $\mathrm{C} \nu \mathrm{B}$ measurement. Thus, it is timely to investigate again the possibility of determining the existence of the $\mathrm{C} \nu \mathrm{B}$ and to study the prospects for determining the neutrino masses via resonant neutrino absorption.

What are the new findings that affect the EHEC $\nu$ absorption probability?

- The original calculation of neutrino absorption was done for a matter-dominated flat universe without a cosmological constant. Recent observations of large-scale gravity, deep-field galaxy counts, and Type Ia supernovae favor a universe with energy fractions $\Omega_{\Lambda} \approx 0.7$ in the cosmological constant and $\Omega_{M} \approx 0.3$ in (mainly cold and dark) matter $[10,11]$. The position of the first Doppler peak in recent $\mathrm{CMB}$ measurements strongly suggests that the universe is flat, i.e. the fractional contribution of curvature energy $\Omega_{k}$ is negligibly 
small. These cosmological parameters, together with the Hubble parameter $H_{0}$, determine the expansion rate of the universe as a function of lookback distance. This expansion history, in turn, crucially impacts the EHEC $\nu$ fluxes at Earth, since their sources are almost certainly located at cosmological distances [83].

- The oscillation interpretation of the atmospheric neutrino data offers a lower limit on the heaviest of the three mass-eigenstates of

$$
m_{\nu_{3}} \geq \sqrt{\triangle m_{\mathrm{atm}}^{2}}>0.04 \mathrm{eV}
$$

at $95 \%$ confidence level (CL), from the inferred mass splitting $\triangle m_{\mathrm{atm}}^{2}$ [84]. On the other hand, studies of the cosmic evolution of the large-scale structure, as observed today, from primordial density perturbations, measured in the $\mathrm{CMB}$, yield an upper bound on the sum of neutrino masses [10-12]

$$
\sum_{i} m_{\nu_{i}} \lesssim 1.2 \mathrm{eV}
$$

Since oscillation studies also reveal that neutrino mass-splittings are small compared to the $\mathrm{eV}$ scale, the cosmic bound per mass-state is conservatively [85] $\sim 0.4 \mathrm{eV}$ - about a factor of five better than the laboratory bounds inferred from tritium beta decay [14, 15], $m_{\nu_{3}}<2.2 \mathrm{eV}(95 \%$ CL) [86], and neutrinoless double beta decay [16], $m_{\nu_{3}} \lesssim(0.66 \div 2.70) \mathrm{eV}[87]$. Thus we have

$$
0.04 \mathrm{eV}<m_{\nu_{3}} \lesssim 0.4 \mathrm{eV} .
$$

It is remarkable that the neutrino mass, whose value was compatible with zero at the time of the original proposal for Z-dips [1], is now known to be not only non-zero, but to lie within a one-order of magnitude range! Accordingly, the resonant annihilation energy for the heaviest mass-state is also known to within an order of magnitude:

$$
1 \times 10^{22} \mathrm{eV} \lesssim E_{\nu_{3}}^{\mathrm{res}}<1 \times 10^{23} \mathrm{eV} .
$$

This resonant energy, when divided by the Z-decay multiplicity of $\sim 40$, predicts secondary cosmic ray particles with energies spanning a decade or more above the Greisen-Zatsepin-Kuzmin (GZK) energy, $E_{\mathrm{GZK}}=4 \times 10^{19} \mathrm{eV}$. This is the energy beyond which the CMB is absorbing to nucleons, due to resonant photopion production [19]. The association of Z-bursts with the mysterious cosmic rays observed above $E_{\mathrm{GZK}}$ is a controversial possibility [20-25]. Intriguingly, the neutrino mass window required in such a scenario coincides quantitatively with Eq. (4) [24].

- Recent proposals for progressively larger EHEC $\nu$ detectors, such as the Pierre Auger Observatory [26], IceCube [27], ANITA [28], EUSO [29],
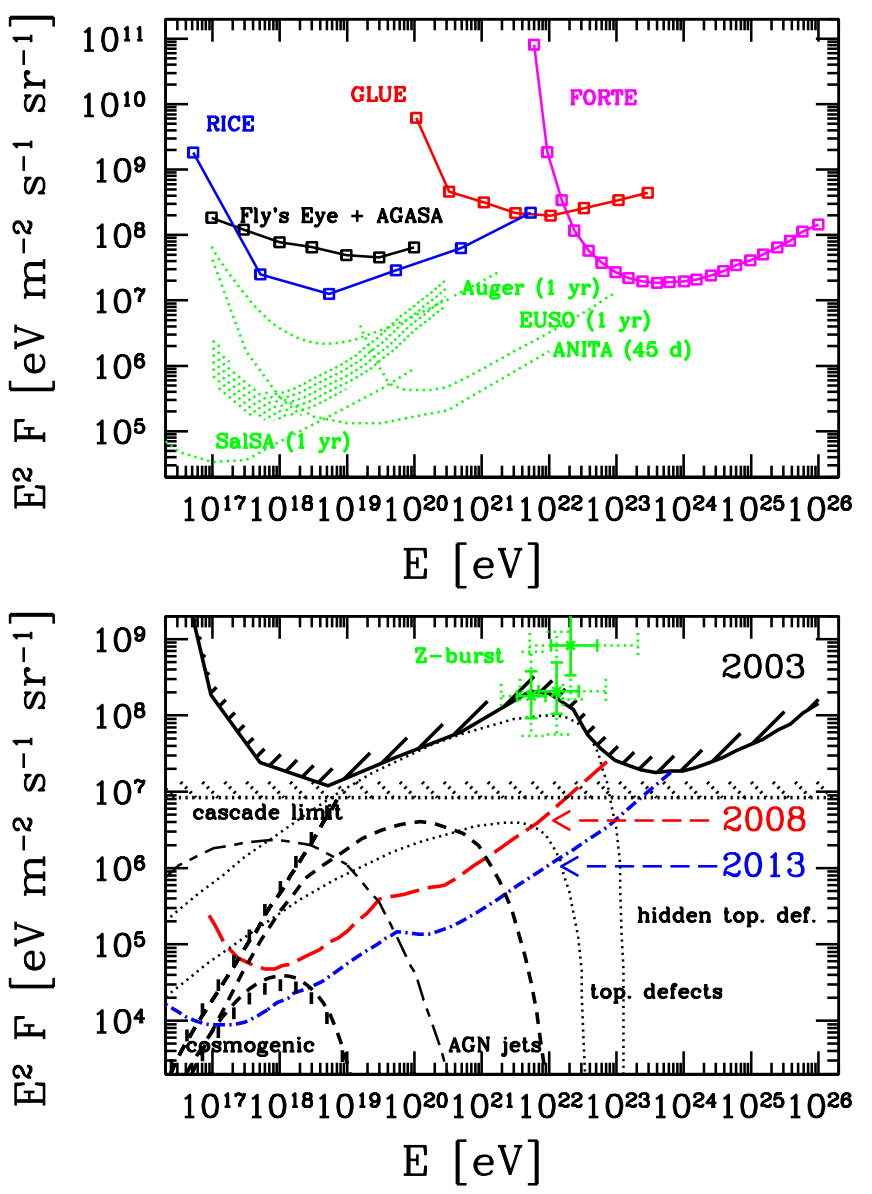

FIG. 1: Current status and next decade prospects for EHEC $\nu$ physics, expressed in terms of diffuse neutrino fluxes per flavor, $F_{\nu_{\alpha}}+F_{\bar{\nu}_{\alpha}}, \alpha=e, \mu, \tau$; full mixing among the flavors en route to Earth [33] is assumed.

Top: Upper limits from RICE [37], GLUE [38], FORTE [39], and Fly's Eye and AGASA [40]. Also shown are projected sensitivities of Auger in $\nu_{e}, \nu_{\mu}$ modes and in $\nu_{\tau}$ mode (bottom swath) [41], ANITA [42], EUSO [43], and SalSA [44], corresponding to one event per energy decade and indicated duration.

Bottom: Roadmap for improvement in the next decade (2008 and 2013; adapted from Ref. [32]), corresponding to one event per energy decade, as well as the current (2003) observational upper bound (solid-shaded) obtained from Fig. 1 (top). For the year 2008 (long-dashed), we assume 3 yr of Auger data and one 15 d ANITA flight. For 2013 (dashed-dotted), we assume 8/3/4 yr Auger/EUSO/SalSA, and 3 ANITA flights. The sensitivity will improve if further projects such as Auger North and OWL [30] are realized, or if the EUSO flight time is extended. Also shown is a wide sample of predictions for $\mathrm{EHEC} \nu$ fluxes (discussed in $\S$ II B).

OWL [30], and SalSA [31] offer credible hope that the collection of an event sample above $10^{21} \mathrm{eV}$ may be realized within this decade [32] (cf. Fig. 1). Another encouraging sign for the progress in experimental sensitivity is that existing $\mathrm{EHEC} \nu$ "observatories", such as RICE [34], GLUE [35], and 
FORTE [36], have recently put the first sensible upper limits on the EHEC $\nu$ flux in the region (5) relevant for neutrino absorption [37-39]. We display these limits in Fig. 1 (top).

The organization of this paper is as follows. In $\S$ II, we determine the $\mathrm{EHEC} \nu$ spectra, with their all-important absorption features, to be observed at Earth. In particular, the depths, widths, and locations of the relic neutrino absorption dips are calculated, for various proposed sources of a diffuse $\mathrm{EHEC} \nu$ flux. The experimental prospects to detect the absorption dips within the next decade or beyond are discussed in $\S$ III. Finally, in $\S$ IV, we summarize our results and conclude.

\section{EHEC $\nu$ SPECTRAL DIPS}

Given an EHEC $\nu$ source emissivity distribution, one can determine the resulting neutrino spectrum to be observed at Earth, by taking into account resonant annihilation with the $\mathrm{C} \nu \mathrm{B}$, and energy losses due to redshift. We formulate this problem, in $\S$ II A, in terms of propagation functions $[45,46]$. We calculate these functions by means of the procedure outlined in the original papers on relic neutrino absorption spectroscopy $[1,2]$, updated to modern cosmological parameters. The observable neutrino flux arriving at Earth is then obtained by folding the propagation function with the $\mathrm{EHEC} \nu$ source emissivity distribution. The latter basic input is not known in the energy region of interest. Therefore, we introduce in $\S$ II B various parameterizations to model neutrino emission from the most relevant classes of possible sources - astrophysical accelerators (bottom-up "Zevatrons") or exotic massive particles and topological defects (top-down). In $\S$ II C, we study the location, depths, and widths of relic neutrino absorption dips in the context of these model classes of sources, and for various neutrino mass scenarios.

\section{A. Neutrino propagation functions}

Let $\mathcal{L}_{\nu_{\beta}}\left(r, E_{i}\right)$ be the EHEC $\nu$ source emissivity distribution, i.e. the number of neutrinos $\nu_{\beta}$ of flavor $\beta=$ $e, \mu, \tau$, per co-moving volume, per unit of time and per unit of energy as measured at the source, injected in the $\mathrm{C} \nu \mathrm{B}$ at a "propagation distance" $r \equiv c t$ from Earth [88] with an energy $E_{i}$. The propagation through the $\mathrm{C} \nu \mathrm{B}$ can be described $[45,46]$ by the functions $P_{b \mid a}\left(E ; E_{i}, r\right)$, which are defined as the expected number of particles of type $b$ above the threshold energy $E$ if one particle of type $a$ started at a distance $r$ with energy $E_{i}$. With the help of these propagation functions, the differential flux of neutrinos of flavor $\alpha\left(b=\nu_{\alpha}\right)$ at Earth, i.e. their number $N_{\nu_{\alpha}}$ arriving at Earth with energy $E$ per units of energy, area $(A)$, time $(t)$ and solid angle $(\Omega)$, can be expressed as

$$
\begin{aligned}
& F_{\nu_{\alpha}}(E) \equiv \frac{\mathrm{d}^{4} N_{\nu_{\alpha}}}{\mathrm{d} E \mathrm{~d} A \mathrm{~d} t \mathrm{~d} \Omega}= \\
& \frac{1}{4 \pi} \int_{0}^{\infty} \mathrm{d} E_{i} \int_{0}^{\infty} \mathrm{d} r \sum_{\beta} \frac{-\partial P_{\nu_{\alpha} \mid \nu_{\beta}}\left(E ; E_{i}, r\right)}{\partial E} \mathcal{L}_{\nu_{\beta}}\left(r, E_{i}\right),
\end{aligned}
$$

where the "propagation distance" $r=c t$ is related to the redshift $z$ by

$$
\mathrm{d} z=(1+z) H(z) \mathrm{d} r,
$$

with the evolving Hubble parameter given by

$$
H^{2}(z)=H_{0}^{2}\left[\Omega_{M}(1+z)^{3}+\Omega_{k}(1+z)^{2}+\Omega_{\Lambda}\right] .
$$

Here, $H_{0}=h 100 \mathrm{~km} / \mathrm{s} / \mathrm{Mpc}$, with $h=(0.71 \pm$ $0.07) \times 1.15$ [5], denotes the present value of the Hubble parameter. In Eq. (8), $\Omega_{M}, \Omega_{k}$, and $\Omega_{\Lambda}$ are the present matter, curvature, and vacuum energy densities, respectively, in terms of the critical density. From the Friedmann equation comes the constraint that fractional energies must sum to $100 \%$, i.e. $\Omega_{M}+\Omega_{k}+\Omega_{\Lambda}=1$. As default values we choose $\Omega_{M}=0.3, \Omega_{k}=0$, and $\Omega_{\Lambda}=0.7$. These values are collectively known as the "concordance" model, for they fit a wide assortment of data [10, 11] [89].

Justified approximations are that (i) the only type of energy loss is due to the redshift, and (ii) the only relevant interaction is absorption on the relic neutrino background, dominated by resonant Z-production [90]. With these approximations, the differential propagation function is given by (see the Appendix for a thorough derivation, properly taking into account neutrino mixing effects)

$$
\begin{aligned}
& \frac{-\partial P_{\nu_{\alpha} \mid \nu_{\beta}}\left(E ; E_{i}, z\right)}{\partial E}= \\
& \delta\left(E-\frac{E_{i}}{1+z}\right) \sum_{j}\left|U_{\alpha j}\right|^{2} P_{\nu_{j}}\left(E_{i}, z\right)\left|U_{\beta j}\right|^{2},
\end{aligned}
$$

where $U_{\alpha j}$ is the leptonic mixing matrix and $P_{\nu_{j}}\left(E_{i}, z\right)$ is the survival probability of a cosmic neutrino $\nu_{j}$ injected at a redshift $z$ with energy $E_{i}=E(1+z)$,

$$
\begin{aligned}
& P_{\nu_{j}}(E(1+z), z)= \\
& \quad \exp \left[-\int_{0}^{z} \frac{\mathrm{d} \tilde{z}}{H(\tilde{z})(1+\tilde{z})} n_{\bar{\nu}_{j}}(\tilde{z}) \sigma_{\nu_{j} \bar{\nu}_{j}}^{\mathrm{ann}}(s)\right],
\end{aligned}
$$

with

$$
s=2 m_{\nu_{j}} E(1+\tilde{z}) .
$$

We remind the careful reader that $z$ is the emission redshift of the cosmic source, while $\tilde{z}$ is the redshift at the time of neutrino annihilation, the latter being integrated 
from present time back to the emission time. The exponent in the brackets is (minus) the optical depth (also called the "opacity") for a neutrino emitted at redshift $z$.

The momentum of a massless neutrino today is of order of the CMB energy, $\sim 3 T_{\gamma} \sim 0.7 \mathrm{meV}$. The neutrino momentum at an earlier epoch is then $\sim 0.7(1+z) \mathrm{meV}$. Thus, relic neutrinos will be non-relativistic as long as $m_{\nu_{j}} \gg\left\langle p_{\nu_{j}}\right\rangle \sim 0.7(1+z)$ meV holds. In most of the region in redshift-space which we consider, the relic neutrinos are non-relativistic. This means that their number densities are given by [5]

$$
\begin{aligned}
n_{\nu_{j}}(\tilde{z})=n_{\bar{\nu}_{j}}(\tilde{z}) & =\left\langle n_{\nu}\right\rangle_{0}(1+\tilde{z})^{3}=\frac{3}{22}\left\langle n_{\gamma}\right\rangle_{0}(1+\tilde{z})^{3} \\
& =56 \mathrm{~cm}^{-3}(1+\tilde{z})^{3}
\end{aligned}
$$

with $\left\langle n_{\nu}\right\rangle_{0}$ and $\left\langle n_{\gamma}\right\rangle_{0}$ denoting todays (subscript " 0 ") av- erage number density in relic neutrinos and relic photons, respectively [91].

The annihilation cross-section $\sigma_{\nu_{j} \bar{\nu}_{j}}^{\text {ann }}(s)$, which is dominated by the Z-production peak with a rather narrowwidth, can be approximated by [1]

$$
\sigma_{\nu_{j} \bar{\nu}_{j}}^{\mathrm{ann}}(s)=\left\langle\sigma_{\nu \bar{\nu}}^{\mathrm{ann}}\right\rangle \delta\left(\frac{s}{m_{Z}^{2}}-1\right)
$$

where

$$
\left\langle\sigma_{\nu \bar{\nu}}^{\mathrm{ann}}\right\rangle=\int \frac{\mathrm{d} s}{m_{Z}^{2}} \sigma_{\nu_{j} \bar{\nu}_{j}}^{\mathrm{ann}}=2 \pi \sqrt{2} G_{F}=40.4 \mathrm{nb}
$$

is the energy averaged cross-section [92] $G_{F}=1.2 \times$ $10^{-5} \mathrm{GeV}^{-2}$ being the Fermi coupling constant [5]. Therefore, the survival probability (10), at the injection energy $E_{i}=E(1+z)$, can be approximated by [1]

$$
\begin{aligned}
P_{\nu_{j}}(E(1+z), z) \simeq \exp & {\left[-\left(\frac{\left\langle n_{\nu}\right\rangle_{0}\left\langle\sigma_{\nu \bar{\nu}}^{\mathrm{ann}}\right\rangle}{H_{0}}\right) \frac{\left(\frac{E_{\nu_{j}}^{\mathrm{res}}}{E}\right)^{3}}{\left.\left[\Omega_{M}\left(\frac{E_{\nu_{j}}^{\mathrm{res}}}{E}\right)^{3}+\Omega_{k}\left(\frac{E_{\nu_{j}}^{\mathrm{res}}}{E}\right)^{2}+\Omega_{\Lambda}\right]^{1 / 2}\right],}\right.} \\
& \text { for } \frac{1}{1+z}<\frac{E}{E_{\nu_{j}}^{\mathrm{res}}}<1,
\end{aligned}
$$

within the region of support indicated, and identically one (no absorption) outside the region of support. Here, $E_{\nu_{j}}^{\mathrm{res}}$ is the resonant energy in the rest system of the relic neutrinos, given in Eq. (1).

Numerically, the annihilation probability on the $\mathrm{C} \nu \mathrm{B}$, neglecting cosmological expansion, is on the few percent level,

$$
\frac{\left\langle n_{\nu}\right\rangle_{0}\left\langle\sigma_{\nu \bar{\nu}}^{\mathrm{ann}}\right\rangle}{H_{0}}=(0.71 / h) \times 3.0 \%
$$

Taking cosmological expansion into account, the annihilation probability is enhanced by the redshift-dependent ratio in the exponent appearing in the expression (15) for the survival probability. The enhancement is easy to understand: In the numerator of the ratio, the factor $\left(E_{\nu_{j}}^{\mathrm{res}} / E\right)^{3}=(1+\tilde{z})^{3}$ accounts for the higher target density of the $\mathrm{C} \nu \mathrm{B}$ in physical volume. The denominator accounts for the time or path length available per unit redshift for annihilation. What is noteworthy here is that, at early times, the $\Omega_{M}$ term dominates, and so the neutrino optical depth scales as $1 / \sqrt{\Omega_{M}}$. Thus, the smaller is $\Omega_{M}$, or equivalently, the larger is $\Omega_{\Lambda}$, the greater is the neutrino absorption probability from sources with $1+z \gtrsim\left(\Omega_{\Lambda} / \Omega_{M}\right)^{1 / 3}$. For example, the $\Lambda$ CDM universe, with $\Omega_{M}=0.3$ and $\Omega_{\Lambda}=0.7$, produces absorption dips nearly twice as deep as a pure CDM universe with $\Omega_{M}=1$. In turn, this alleviates the statistics requirement (discussed later) by a factor of $\sim 3$.

In Fig. 2, we display the survival probability (15) for the modern concordance cosmological parameters, i.e. $\Omega_{M}=0.3, \Omega_{k}=0, \Omega_{\Lambda}=0.7$, and $h=0.71$ (top), and their allowed variations (bottom), respectively. It seems that variations of $\Omega_{M}, \Omega_{\Lambda}$, and $h$, within their uncertainties $[10,11]$, amount to a $\sim 5 \%$ effect.

As is apparent from Fig. 2 (top), using the narrowwidth approximation (13) for the annihilation crosssection rather than taking into account the complete energy dependence of the cross-section is justified within the overall $5 \%$ error.

\section{B. Neutrino source emissivity distributions}

In the previous subsection, we have found that, for a given source emissivity distribution $\mathcal{L}_{\nu_{\beta}}$ of neutrinos of flavor $\beta$, the neutrino flux of flavor $\alpha$ to be observed at 

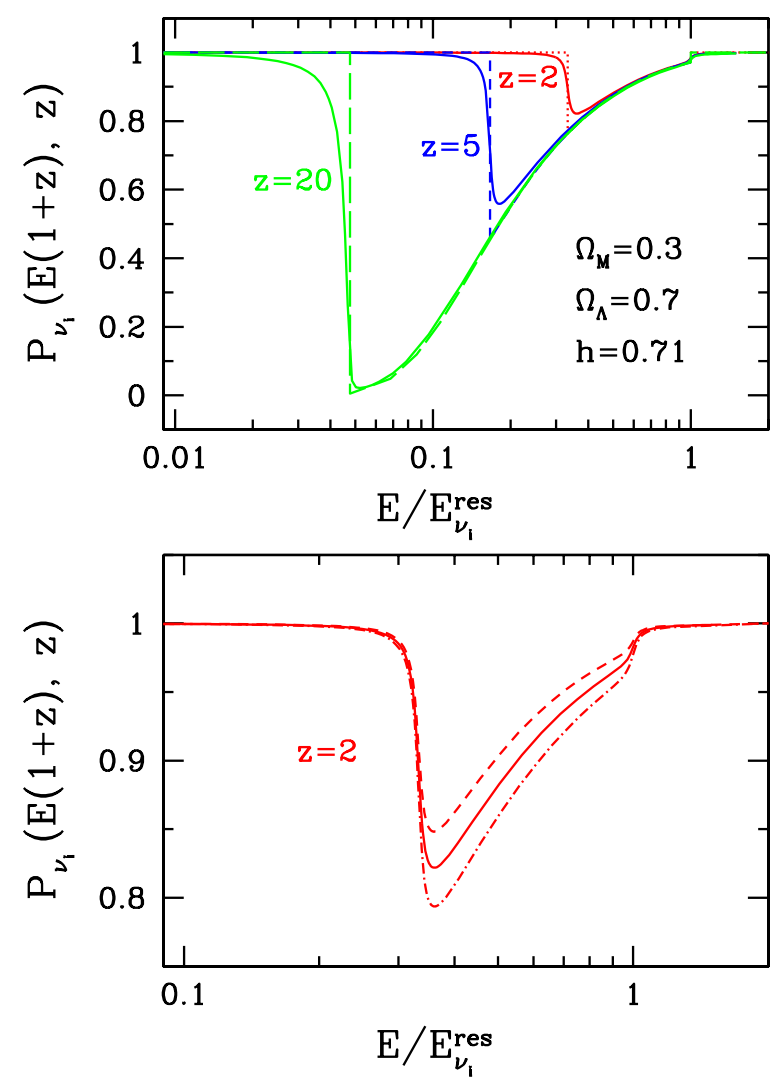

FIG. 2: The survival probability $P_{\nu_{i}}\left(E_{i}, z\right)$ of a cosmic neutrino $\nu_{i}$, injected at redshift $z$ with energy $E_{i}$, as a function of the energy at Earth, $E=E_{i} /(1+z)$, in units of the resonance energy $E_{\nu_{i}}^{\text {res }}=m_{Z}^{2} / 2 m_{\nu_{i}}$.

Top: The narrow-width approximation (15), for $z=2$ (dotted), $z=5$ (short-dashed), $z=20$ (long-dashed), and standard cosmological parameters $\left(\Omega_{M}=0.3, \Omega_{\Lambda}=0.7\right.$, $h=0.71)$, compared with the complete energy dependence from the annihilation cross-section of Ref. [3] (solid).

Bottom: The survival probability for $z=2$ and standard cosmological parameters (solid) compared with the most extreme variations allowed by up-to-date global fits: $\Omega_{M}=0.20$, $\Omega_{\Lambda}=0.78, h=0.81$ (dashed) and $\Omega_{M}=0.40, \Omega_{\Lambda}=0.61$, $h=0.62$ (dashed-dotted) [11].

Earth is predicted to be (cf. Eqs. (6) - (9))

$$
\begin{aligned}
& F_{\nu_{\alpha}}(E)=\frac{1}{4 \pi} \int_{0}^{\infty} \frac{\mathrm{d} z}{H(z)} \times \\
& \sum_{\beta, j}\left|U_{\alpha j}\right|^{2} P_{\nu_{j}}(E(1+z), z)\left|U_{\beta j}\right|^{2} \mathcal{L}_{\nu_{\beta}}(z, E(1+z)),
\end{aligned}
$$

where $\mathcal{L}_{\nu_{\beta}}(z, E(1+z))$ is the number of neutrinos of flavor $\beta$ and energy $E_{i}=E(1+z)$ emitted per co-moving volume, per unit time and unit energy, at a redshift "distance" $z$ (cf. Eq. (7)). In this subsection and the one that follows, we evaluate this expression further for some benchmark source emissivities.
We will focus mainly on sources which produce pions ("hadronic" sources), be they astrophysical Zevatrons (bottom-up) or non-accelerator (top-down) ones. From the decay sequence $\pi^{ \pm} \rightarrow \mu^{ \pm}+\nu_{\mu} \rightarrow e^{ \pm}+2 \nu_{\mu}+\nu_{e}$ (neither we, nor experiments, will distinguish neutrinos from anti-neutrinos), the flavor ratios of the source emissivities are predicted to be

$$
\mathcal{L}_{\nu_{e}}: \mathcal{L}_{\nu_{\mu}}: \mathcal{L}_{\nu_{\tau}}=1: 2: 0
$$

In this case, one finds - exploiting the fact that phenomenologically $\left|U_{e 3}\right|^{2} \ll 1$ and $\left|U_{\mu 3}\right| \simeq\left|U_{\tau 3}\right|$ - that the fluxes at Earth are well approximated by (see Appendix and also Ref. [33])

$$
\begin{aligned}
F_{\nu_{\alpha}}(E) \simeq \frac{1}{4 \pi} & \int_{0}^{\infty} \frac{\mathrm{d} z}{H(z)} \frac{1}{3} \mathcal{L}_{\nu}^{\mathrm{tot}}(z, E(1+z)) \\
& \times \sum_{j=1}^{3}\left|U_{\alpha j}\right|^{2} P_{\nu_{j}}(E(1+z), z),
\end{aligned}
$$

where $\mathcal{L}_{\nu}^{\text {tot }}$ is the total, flavor-summed neutrino emissivity at the source. In fact, as discussed in the Appendix, Eq. (19) holds whenever the source emissivities satisfy $\mathcal{L}_{\nu_{\mu}}+\mathcal{L}_{\nu_{\tau}}=2 \mathcal{L}_{\nu_{e}}$. Thus, it holds also for a "democratic" flavor ratio of source emissivities,

$$
\mathcal{L}_{\nu_{e}}: \mathcal{L}_{\nu_{\mu}}: \mathcal{L}_{\nu_{\tau}}=1: 1: 1
$$

as might arise from the decay of topological defects not coupled directly to ordinary matter such as, for example, mirror-matter "necklaces" [50].

It is unlikely that neutrino observatories will be able to distinguish neutrino flavors at extreme high-energy [51]. Phenomenologically then, we are mainly interested in the sum over all neutrino flavors $\alpha$. With such a sum, unitarity completely removes the dependence on the leptonic mixing matrix from Eq. (19), leaving just

$$
\begin{aligned}
\sum_{\alpha} F_{\nu_{\alpha}}(E) \simeq \frac{1}{4 \pi} & \int_{0}^{\infty} \frac{\mathrm{d} z}{H(z)} \frac{1}{3} \mathcal{L}_{\nu}^{\mathrm{tot}}(z, E(1+z)) \\
& \times \sum_{j=1}^{3} P_{\nu_{j}}(E(1+z), z) .
\end{aligned}
$$

Another simplification of Eq. (19) occurs if the neutrino masses are quasi-degenerate, which in fact is realized in Nature if the lightest neutrino has a mass $m_{\nu_{1}} \gg \triangle m_{\text {atm }}^{2}$, say, $m_{\nu_{1}} \gtrsim 0.1 \mathrm{eV}$ (cf. Fig. 3):

$$
m_{\nu_{1}}: m_{\nu_{2}}: m_{\nu_{3}} \simeq 1: 1: 1 \Rightarrow P_{\nu_{1}} \simeq P_{\nu_{2}} \simeq P_{\nu_{3}} .
$$

With quasi mass-degeneracy, Eq. (19) simplifies to

$$
\begin{aligned}
& F_{\nu_{\alpha}}(E) \simeq \\
& \frac{1}{4 \pi} \int_{0}^{\infty} \frac{\mathrm{d} z}{H(z)} P_{\nu_{1}}(E(1+z), z) \frac{1}{3} \mathcal{L}_{\nu}^{\mathrm{tot}}(z, E(1+z))
\end{aligned}
$$



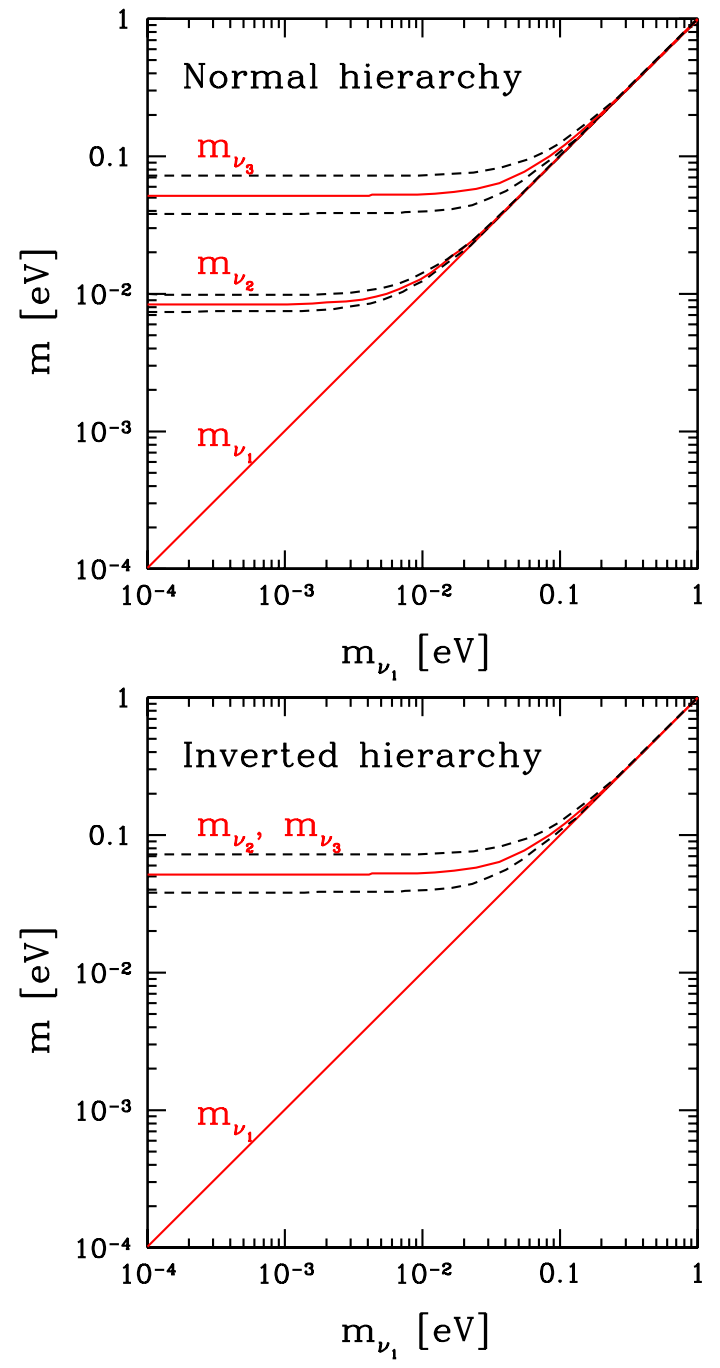

FIG. 3: Allowed ranges for the neutrino masses as a function of the lightest neutrino mass $m_{\nu_{1}}$, in the normal (top) and inverted (bottom) three-neutrino scheme (adapted from Refs. [52, 53]).

for each $\alpha=e, \mu, \tau$.

In the following, we assume that the $\operatorname{EHEC} \nu$ sources, which build up the diffuse source emissivity, have identical injection spectra $J_{\nu_{\beta}}$. Consequently, the $z$ and $E_{i}$ dependences of the source emissivity distribution factorize,

$$
\mathcal{L}_{\nu_{\beta}}\left(z, E_{i}\right)=\eta(z) J_{\nu_{\beta}}\left(E_{i}\right) .
$$

The "activity" $\eta(z)$ is the number of sources per comoving volume and per unit of time, at the redshift "time" $z$, while the injection spectra $J_{\nu_{\beta}}\left(E_{i}\right)$ are the number of neutrinos $\nu_{\beta}$ emitted by a single source per unit of energy. The $z$-dependence of the activity accounts for any evolution of the co-moving number density and/or of the common luminosity of the sources.

We employ several parameterizations of $\eta(z)$, which allow us to study a variety of possible EHEC $\nu$ origins - ranging from astrophysical accelerator sources such as gamma ray bursts (GRB's) and active galactic nuclei (AGN's) which turned on at $z \sim$ few, to non-accelerator sources such as topological defects which have been decaying all the way back to $z$ very large.

We start with a parametrization of the activity motivated by astrophysics [54],

$$
\eta_{\mathrm{SFR}}(z)=\eta_{0} \frac{1+a}{(1+z)^{-n_{1}}+a(1+z)^{n_{2}}},
$$

with $\eta_{0}=\eta(z=0)$ being the activity in the today's epoch. With the values $a=0.005(0.0001), n_{1}=3.3(4.0)$, and $n_{2}=3.0(3.0)$, the parametrization fits the star formation rate (SFR) history derived from the blue and UV luminosity density, in line with the extreme ranges of optical/UV measurements without [55] (with [56]) dust extinction corrections. We will refer to these two cases as conservative and generous SFR, respectively. The star formation rate is believed to map out the earliest structures sufficiently bound by gravity to undergo fusion. As such, they may map out the history of AGN and GRB evolution, two potential sources of the EHEC rays (EHECR's) [54].

The parametrization (25) provides a peak activity at

$$
1+z_{\text {peak }}=\left(\frac{n_{1}}{a n_{2}}\right)^{\frac{1}{n_{1}+n_{2}}}
$$

which occurs at $z_{\text {peak }}=1.4$ for the conservative SFR and at $z_{\text {peak }}=2.9$ for the generous one. For $n_{2}=0, a=0$ it reduces to a simple power-law, $\eta(z)=\eta_{0}(1+z)^{n_{1}}$. Such a choice, with $n_{1} \sim 4$ to fit the low- $z$ SFR, is often used in the literature. Without a cutoff in $z$, however, the power-law provides an extreme evolution history, as the activity increases indefinitely.

This brings us to a further parametrization, namely a simple power-law ansatz, but with cutoffs $z_{\min }$ and $z_{\max }$ to exclude the existence of nearby and early-time sources, respectively:

$$
\eta_{\text {pow }}(z)=\eta_{0}(1+z)^{n} \theta\left(z-z_{\min }\right) \theta\left(z_{\max }-z\right) .
$$

This ansatz has the advantage of leading to easily tractable analytic expressions, while still reproducing, for $n=4$, the generous SFR case, as long as $z_{\max }<z_{\text {peak }}$. In addition to describing the evolution of GRB's and AGN's up to $z \sim 2$, the power-law with $n \simeq 1 \div 2$ and $z_{\max } \gg 1$ also characterizes the activity expected from non-accelerator sources. For example, topological defect sources [57] are characterized by $n=3 / 2$ [93] and $z_{\max }$ arbitrarily large [94]. Throughout, we will take $z_{\text {min }}=0$ as a default value [95].

Turning to the source injection spectra $J_{\nu_{\beta}}\left(E_{i}\right)$, we only need to specify them in the energy decade around the respective resonance energies. For this energy decade, we again assume a power-law behavior [96],

$$
J_{\nu_{\beta}}\left(E_{i}\right)=j_{\nu_{\beta}} E_{i}^{-\alpha} \theta\left(E_{i}-E_{\min }\right) \theta\left(E_{\max }-E_{i}\right) .
$$


As with the activity power-law, this spectral power-law parametrization facilitates the analytic study of different scenarios for the origin of EHEC $\nu$ 's.

In most of the source models, the neutrinos originate from pion decays, the latter either being produced in inelastic $p p$ or $p \gamma$ interactions (astrophysical sources), or, alternatively, arising in the fragmentation of QCD jets in the decays of superheavy particles (top-down sources). Although the resulting neutrino spectra $J_{\nu_{\beta}}$ can be fairly well calculated for given injection spectra of protons or pions, the details are tedious and not worth the effort for our present purposes, and so we retain the simple parametrization (28) instead. The power-law should mimic the various neutrino injection spectra reasonably well, with the spectral index in the range $\alpha \simeq 1 \div 2$. Throughout, we will take $E_{\min }=0$ as a default value.

For astrophysical accelerators, one expects that $E_{\max }$ is a fraction $(\sim 5 \%)$ of the maximum proton energy, $E_{p \max }$. In the case of shock acceleration, $E_{p \max }$ is determined by the requirement that the gyromagnetic radius of the protons in the ambient magnetic field $B$ be less than the spatial extension $R$ of the accelerating source. The result is

$$
E_{p \max }^{\text {shock }} \simeq 10^{21} \mathrm{eV}(R / \mathrm{kpc})(B / \mathrm{mG}) .
$$

Even higher energies are possible in proposed nonshock mechanisms, such as unipolar induction, acceleration in strong electromagnetic waves in plasmas (wakefields) [58], or by magnetic recombination in the vicinity of massive black holes [59].

For top-down scenarios, $E_{\max }$ can be much larger, basically bounded only by the huge mass of the decaying particle or defect. These huge masses are thought to reflect the energy-scale of an underlying phase transition. Popular examples include $M_{\mathrm{GUT}} \sim 10^{16} \mathrm{GeV}$ from grand-unification, and $M_{\text {wimpzilla }} \sim 10^{11 \div 13} \mathrm{GeV}$ from the end of inflation. Neutrinos from the decay of these super-massive objects carry an energy about one order of magnitude less than the mass.

In general, there may be several classes of sources such as GRB's, AGN's, EHEC protons scattering inelastically off the CMB ("cosmogenic" neutrinos), and topological defects - which build up the total emissivity distribution. Predictions from a variety of such source classes are shown in Fig. 1 (bottom): from jets of AGN's [60], from ordinary topological defects $\left(M_{X}=\right.$ $\left.10^{14} \mathrm{GeV}\right)$ [61], from hidden-sector topological defects $\left(M_{X}=4 \times 10^{14} \mathrm{GeV}\right)$ [62], and from the Z-burst scenario normalized to fit the highest energy cosmic ray anomaly [24] with different assumptions about the universal radio background. Also shown are the upper and lower bounds [46] (short-dashed-shaded) and one example [61] (short-dashed) of the cosmogenic neutrino flux. Finally, the cascade limit [63] from Ref. [64] (dottedshaded) on transparent neutrino sources (discussed later) is shown. It applies to all scenarios where neutrinos originate from pion decays or even from electroweak jets [65]. These neutrinos are accompanied by photons and elec- trons which cascade down in energy during their propagation through the universe. The cascade limit arises from the requirement that the associated diffuse gammaray fluxes should not exceed measurements [97].

It is straightforward to generalize Eq. (24) to a sum of source classes. In reality, it is more prudent to wish for even one class of sources at the energies of interest here, $10^{22 \div 23} \mathrm{eV}$, and so we continue to work within the single source-class hypothesis.

Another possibility, in principle, is to collect events from a small number of point sources, possibly just one. This presents the advantage that the spectra are not smeared by the integration over the redshift distribution. Since neutrinos are not deflected in flight, pointsource selection is possible. However, we will find that the event numbers required for statistical significance is on the order of 100 or more. This large number presents a luminosity challenge for a small number of very bright sources [2]. In the end, it does not matter much for our purposes whether the $\mathrm{EEC} \nu$ flux is diffuse of granular, since the isotropy of the relic background ensures nearly the same absorption shapes in the spectrum of either source-type.

\section{Case studies}

Let us start our analyses with the quasi-degenerate neutrino mass-spectrum case, whose implications are summarized in Eq. (22). In view of the expectation that $\mathrm{EHEC} \nu$ neutrino fluxes are rapidly falling with energy (cf. Fig. 1), this case has the best prospects of observability, since it corresponds to the largest neutrino masses (cf. Fig. 3), and it brings all three resonance energies to a common lowest value (1). As discussed above (cf. Eq. (23)), we expect in this case an identical absorption dip in the neutrino flux of each flavor to be observed at Earth, if the flavor ratios at the sources were hadronlike (18) or democratic (20).

In Fig. 4, we show the predicted flux $F_{\nu_{\alpha}}$ for a hadronic or flavor democratic source emissivity characterized by a power-law activity (27) with redshift evolution index $n$, and power-law injection spectrum (28) with index $\alpha$. This flux is normalized to the predicted flux for no absorption, $F_{\nu_{\alpha}}^{\text {no abs }}$ (obtained by replacing the survival probability in Eq. (23) by unity). A nice feature following from the two power-laws, source activity and source emissivity, is that the normalized spectrum depends on $n$ and $\alpha$ only through the combination $n-\alpha$; the source evolution and the energy fall-off compensate each other in a simple way. Three particular $n-\alpha$ combinations are shown in Fig. 4 for each fixed $z_{\max }: n-\alpha=0$ (upper curves), $n-\alpha=2$ (middle curves), and $n-\alpha=4$ (lower curves).

Figure 4 illustrates some general features. Viewed as a function of decreasing energy, the absorption dip starts abruptly at the resonance energy $E_{\nu_{i}}^{\text {res }}$. This initial depth is determined by today's annihilation probability, $\approx 3 \%$ 


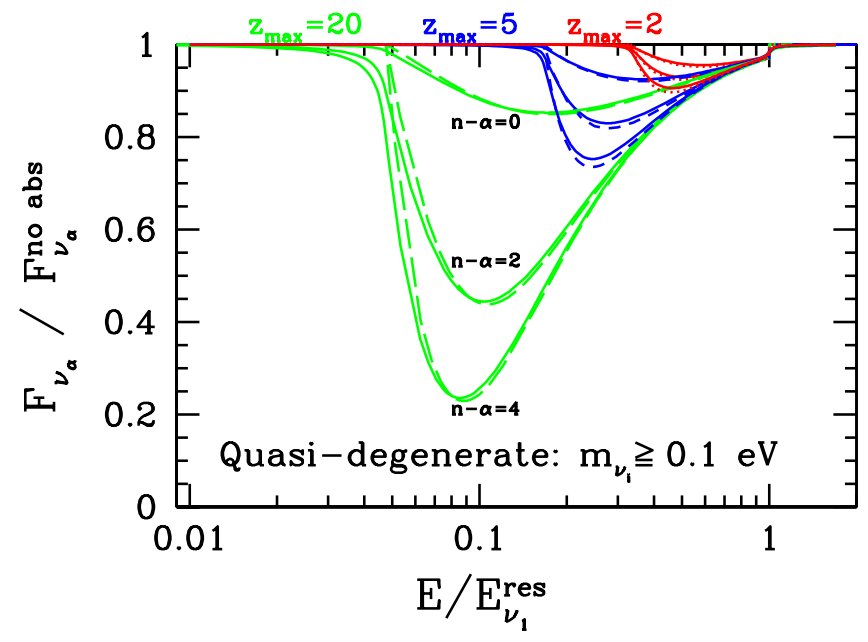

FIG. 4: Predicted flux (23) of neutrinos $\nu_{\alpha}$ of flavor $\alpha=e, \mu, \tau$ at Earth, for a source emissivity characterized by a power-law activity (27) and a power-law injection spectrum (28), for the case of quasi-degenerate neutrino masses (22), normalized to the predicted flux for no absorption. $E / E_{\nu}^{\text {res }}$ scales as the degenerate mass $m_{\nu}$. Three values of $z_{\max }$ are shown: 2 (dotted), 5 (short-dashed), and 20 (long-dashed). For each choice of $z_{\max }$, three choices of $n-\alpha$ are shown: 0 (upper), 2 (middle), and 4 (lower). The corresponding solid lines show the same quantity evaluated with the complete energy dependence of the annihilation cross-section from Ref. [3] arising from the finite $\mathrm{Z}$-width, instead of exploiting the zero-width approximation (13). For all curves, $E_{\max }>E_{\nu_{1}}^{\text {res }}\left(1+z_{\max }\right)$ is assumed.

according to (16), and reflects the absorptions occurring in nearby $(z \sim 0)$ space. The dip extends in energy down to $E_{\nu_{i}}^{\text {res }} /\left(1+z_{\max }\right)$, with this minimum energy being the redshifted value of the resonant energy for annihilations occurring at cosmic distance $\left(z \sim z_{\max }\right)$. The overall depth of the dip increases dramatically with $n-\alpha$, thereby showing a strong preference for source evolution and/or flat energy-spectra. With increasing $z \sim z_{\max }=2,5,20$, the absorption depths are roughly 5 , 8 , and $15 \%$ for $n-\alpha=0 ; 7,18$, and $55 \%$ for $n-\alpha=2$; and 10,27 , and $77 \%$ for $n-\alpha=4$, respectively. We observe in Fig. 4, as we did in Fig. 2 (top), that the replacement of the finite Z-width with the $\delta$-function approximation (13) is well justified for our purposes. From now on, we will exploit this simplification.

Next, we consider some non-degenerate neutrino mass scenarios, and source activities other than power-law. In the four panels of Figs. $5-8$, we show the prediction (21) for $\sum_{\alpha} F_{\nu_{\alpha}} / \sum_{\alpha} F_{\nu_{\alpha}}^{\text {no abs }}$ for a neutrino spectrum which is quasi-degenerate, i.e. lightest mass $m_{\nu_{1}}=0.4 \mathrm{eV}$ (upper panel), normal hierarchical, with $m_{\nu_{1}}=0.01 \mathrm{eV}$ (2nd panel) and $m_{\nu_{1}}=0.002 \mathrm{eV}$ (3rd panel), and inverted hierarchical with $m_{\nu_{1}}=0.002 \mathrm{eV}$ (bottom panel). Figures 5 and 6 display results for $z_{\max }=10, \alpha=2$ (solid) and 0 (dashed), and the conservative and generous starformation activities, respectively. Figure 7 shows results for a power-law activity, $n-\alpha=0$, and $z_{\max }=10$ (shortdashed) and 20 (solid), mimicking a topological defect source scenario. Fig. 8 displays results for a power-law activity, $n-\alpha=2$, and $z_{\max }=2,5,10$ from upper to lower curves, corresponding to bottom-up acceleration. As expected, the depths of the dips increase markedly with $z_{\max }$, lower spectral index $\alpha(28)$, and increased source evolution (27), the latter represented either by the shift from "conservative" to "generous" SFR as in Figs. 5 and 6 , or by the increase in $n-\alpha$ as in Figs. 7 and 8.

The 2nd panels in the figures illustrate that in the case of a normal hierarchy and an intermediate lightest neutrino mass of $m_{\nu_{1}}=0.01 \mathrm{eV}$, corresponding to central values $m_{\nu_{2}}=0.013 \mathrm{eV}$ and $m_{\nu_{3}}=0.053 \mathrm{eV}$ in Fig. 3 (top), the absorption dips from $\nu_{1}$ and $\nu_{2}$ cannot be resolved. The two dips appear as one dip, which is understandably about twice as deep as the single dip from $\nu_{3}$.

The two lower panels present results for a very small, lightest neutrino mass of $m_{\nu_{1}}=0.002 \mathrm{eV}$. In the normal hierarchical case (3rd panel), three absorption dips - associated with the masses $m_{\nu_{1}}=0.002 \mathrm{eV}$, and, from the central values of Fig. 3 (top), $m_{\nu_{2}}=0.0085 \mathrm{eV}$ and $m_{\nu_{3}}=0.052 \mathrm{eV}$ - are clearly visible. These 3rd panels present relic neutrino mass absorption spectroscopy at its best! For an inverted hierarchy, on the other hand, one finds, as illustrated in the bottom panels, that the absorption dips from $\nu_{2}$ and $\nu_{3}$ cannot be resolved individually. With $m_{\nu_{1}}=0.002 \mathrm{eV}$, the central values for the nearly-degenerate heavy masses are $m_{\nu_{2}} \simeq m_{\nu_{3}}=0.052 \mathrm{eV}$, from Fig. 3 (bottom).

The $2 \mathrm{meV}$ mass is a critical one in the sense that the relic neutrino $\nu_{1}$ is indeed non-relativistic for $z$ up to $z_{\max }=2$, in which case Eq. (12) is applicable. For even smaller masses (or larger $z_{\text {max }}$ ), the relic $\nu_{1}$ density becomes relativistic and the absorption dip is known to wash out [1]. Thus, the two lower panels in Figs. 5-8 remain applicable for any $m_{\nu_{1}}<0.002 \mathrm{eV}$, if one simply removes the contribution of the highest-energy dip, at $\sim 10^{24} \mathrm{eV}$.

\section{EXPERIMENTAL PROSPECTS}

In the last paragraph we have seen that, depending on the source activity, injection spectrum, and neutrino masses, we may expect absorption dips of $(10 \div 20) \%$ depth, located at $(0.1 \div 0.5) E_{\nu_{i}}^{\text {res }}$, with a width of about an order of magnitude in energy. Is there any hope of discovery of these absorption dips in the next decade or beyond? The answer to this question depends critically on the magnitude of the EHEC $\nu$ flux at the resonance energies realized in Nature. In the following we will study a few benchmark flux scenarios ( $§$ III A) and discuss further experimental ( $\S$ III B) issues concerning the clean reconstruction of absorption dips. 

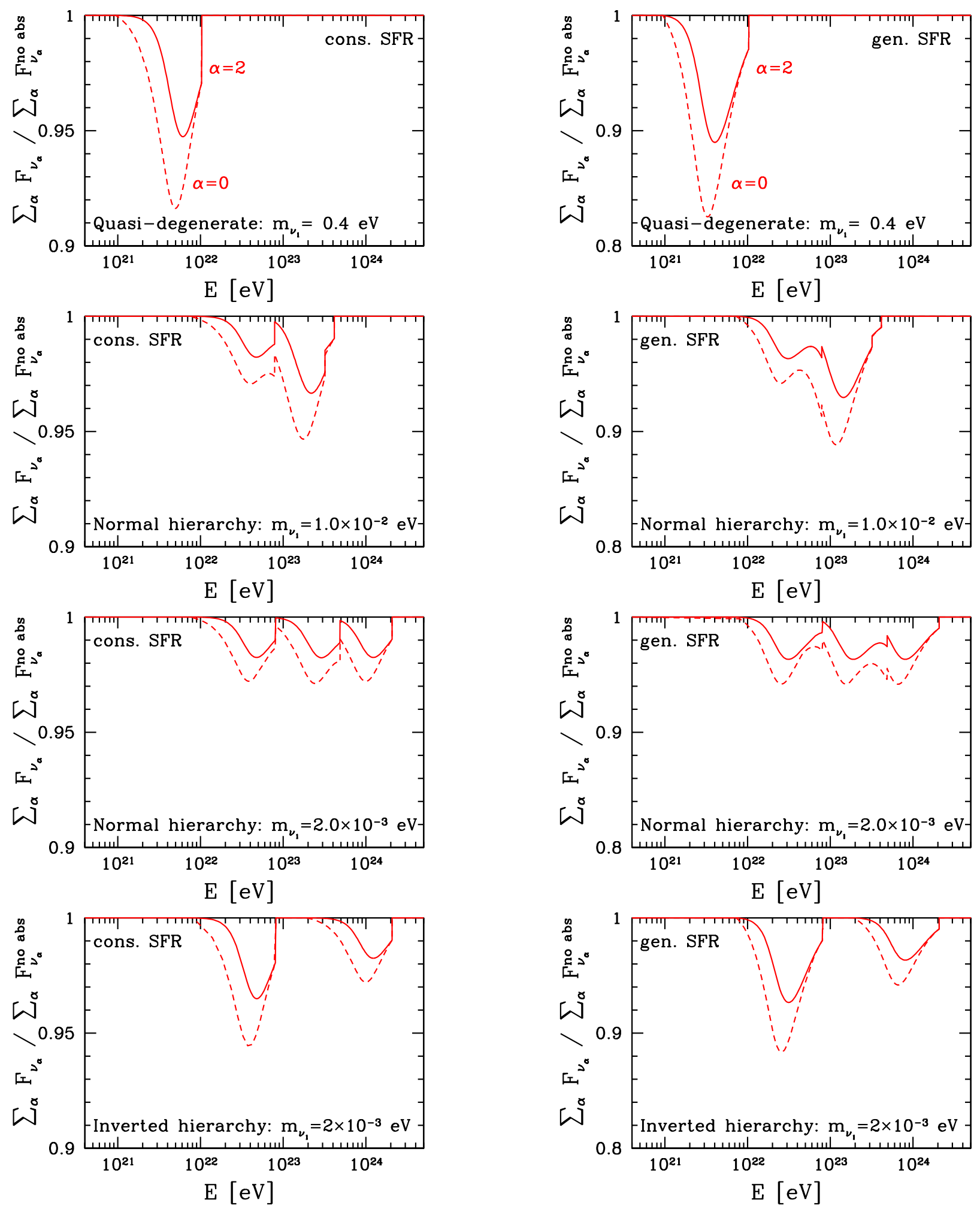

FIG. 5: Predicted flux of neutrinos summed over flavors at Earth (21), normalized to the predicted flux for no absorption, for a conservative SFR activity (25) to $z_{\max }=10$, injectionspectrum indices $\alpha=2$ (solid) and $\alpha=0$ (dashed) (28), and neutrino spectra which are quasi-degenerate (top), normalhierarchical (2nd and 3rd panels), and inverted-hierarchical (bottom panel). For all curves it is assumed that $E_{\max }>$ $E_{\nu_{1}}^{\text {res }}\left(1+z_{\max }\right)$.

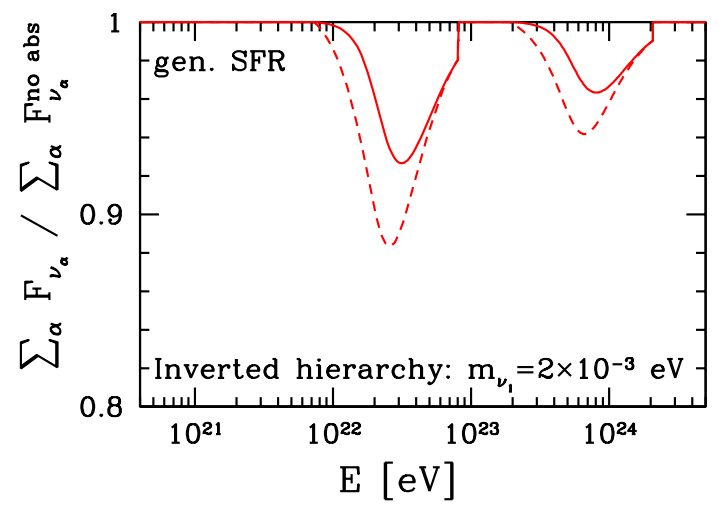

FIG. 6: Same as Fig. 5, but with the generous SFR activity (25). 

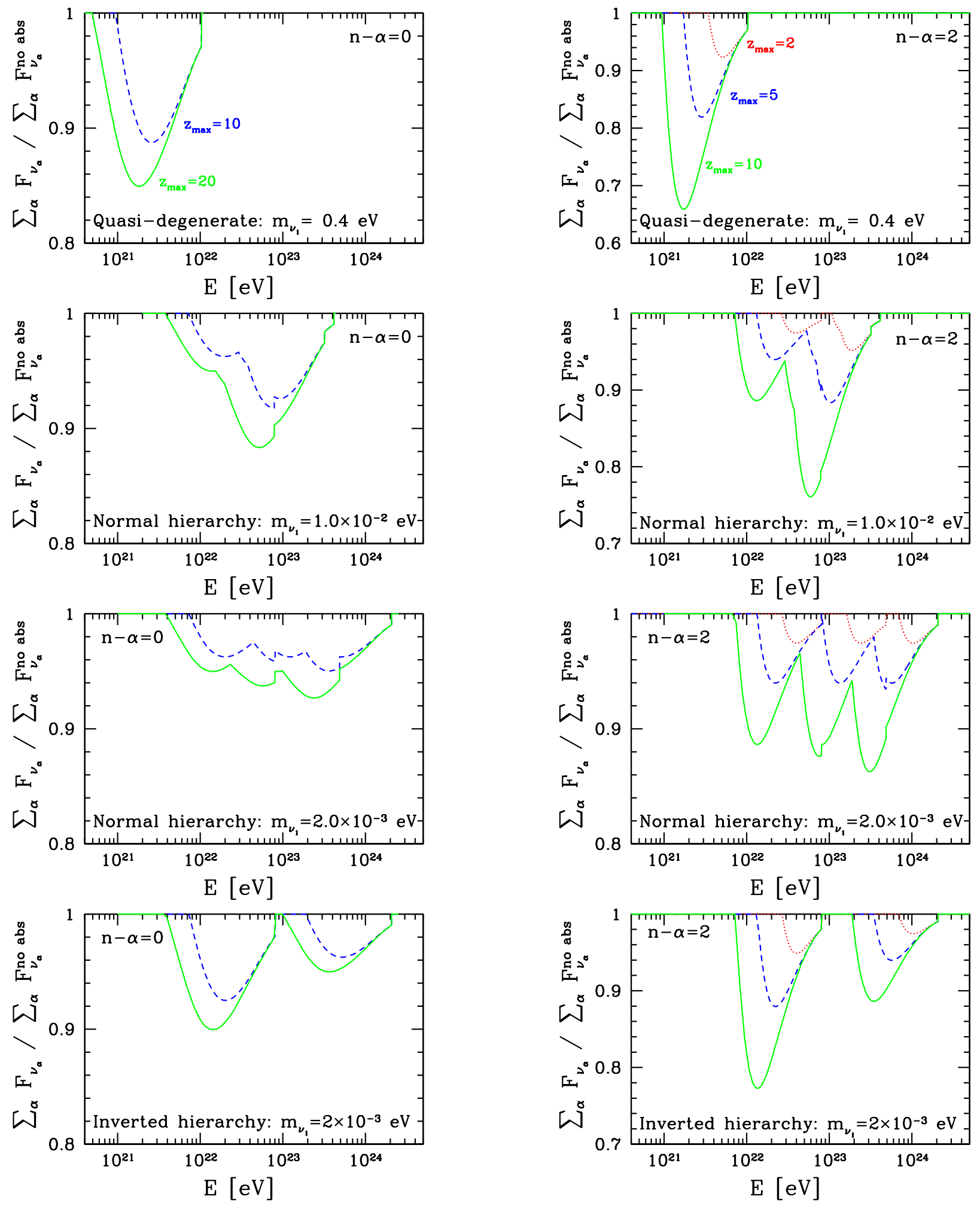

FIG. 7: As in Fig. 5, but with a power-law activity (27) and $n-\alpha=0$, with $z_{\min }=0$ and $z_{\max }=10$ (short-dashed), 20 (solid), mimicking a topological defect source scenario. For all curves it is assumed that $E_{\max }>E_{\nu_{1}}^{\mathrm{res}}\left(1+z_{\max }\right)$.

FIG. 8: Same is Fig. 7, but with $n-\alpha=2$ fixed, and $z_{\max }=2,5,10$ (from upper to lower curves), corresponding to a bottom-up acceleration source scenario. 
TABLE I: Expected number of neutrinos (plus anti-neutrinos) to be detected in upcoming EHEC $\nu$ observatories with energies in the indicated intervals until the indicated year, for two different $\mathrm{EHEC} \nu$ flux scenarios - one saturating the current observational upper bound and one saturating the cascade limit (cf. Fig. 1 (bottom)).

\begin{tabular}{|l||r|r|r|r|r|r|}
\hline \hline \multicolumn{1}{|c||}{} & \multicolumn{5}{c|}{$\sum_{\alpha} \triangle\left(N_{\nu_{\alpha}}+N_{\bar{\nu}_{\alpha}}\right)$} \\
\hline energy decade & $10^{21 \div 22} \mathrm{eV}$ & $10^{22 \div 23} \mathrm{eV}$ & \multicolumn{2}{|c|}{$10^{23 \div 24} \mathrm{eV}$} \\
\hline year & 2008 & 2013 & 2008 & 2013 & 2008 & 2013 \\
\hline \hline observ. limit & 240 & 700 & 30 & 90 & 2 & 5 \\
\hline cascade limit & 13 & 40 & 3 & 10 & 1 & 2 \\
\hline \hline
\end{tabular}

\section{A. Benchmark flux scenarios}

\section{Most optimistic scenario: hidden sources}

The most favorable case for relic neutrino absorption spectroscopy is the one in which the neutrino fluxes saturate the current observational upper bounds. It is known quantitatively (cf. the points marked "Z-burst" from Ref. [24] in Fig. 1 (bottom)) that in this most favorable flux scenario, the secondary fluxes of protons (and photons) from hadronic Z-decay are remarkably of just the right order of magnitude to explain the highest energy cosmic rays above the GZK energy by Z-bursts [20, 22].

As can be seen from Fig. 1 (bottom) and as summarized in Table I, the upcoming EHEC $\nu$ observatories expect to see in this case in 2013 about 230 neutrinos (plus anti-neutrinos) per flavor $\alpha=e, \mu, \tau$ in the energy interval from $10^{21} \mathrm{eV}$ to $10^{22} \mathrm{eV}$. The total number of neutrino events, then, is $N \simeq 700$ neutrinos of all flavors, which implies a 1-sigma fluctuation of $\sqrt{N} \simeq 26$. For a 3 -sigma evidence for an absorption dip in this energy interval, an absorption depth of $3 \sqrt{N} / N \simeq 11 \%$ is required; for a 5 sigma discovery, a depth of $5 \sqrt{N} / N \simeq 19 \%$ is required.

As a comparison with our case studies in $\S$ II C reveals, these depth requirements are achievable as long as the dip is maximized via a quasi-degenerate neutrino mass. The quasi-degenerate condition is $m_{\nu_{1}} \gtrsim 0.1 \mathrm{eV}$, which in turn implies that $E_{\nu_{i}}^{\text {res }} \lesssim 4 \times 10^{22} \mathrm{eV}$. For example, power-law source emissivities produce such depths for $n-\alpha \gtrsim 0$ and/or $z_{\max } \gtrsim 10$ (cf. Figs. 4, 7 (top), and 8 (top)). Therefore, the realization of the Z-burst mechanism for the already observed highest energy cosmic ray events implies not only a discovery of the corresponding $\mathrm{EHEC} \nu$ flux by 2008 (cf. Table I), but also the discovery of solid evidence for the associated absorption dip as soon as 2013.

What kind of source could produce such an optimistic $\mathrm{EHEC} \nu$ flux, of the order of the observational limit at $10^{21} \mathrm{eV}$ to $10^{22} \mathrm{eV}$ ? Bottom-up astrophysical Zevatrons may in principle produce such a flux, with the problem that they must accelerate protons to ener-

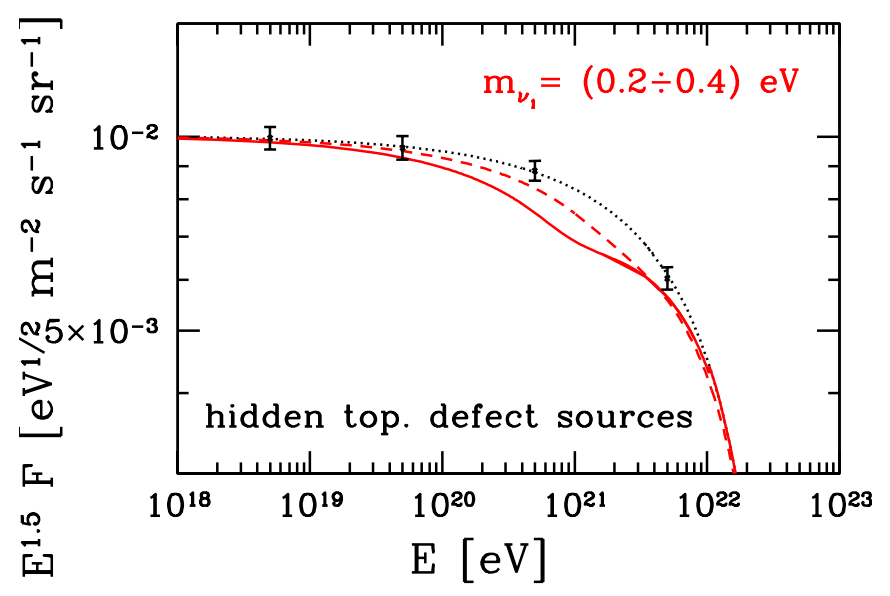

FIG. 9: Predicted neutrino flux at Earth, summed over all flavors, from a power-like source emissivity, with $n=1.5$, $z_{\max }=\infty, \alpha=1.5$, and $E_{\max }=4 \times 10^{22} \mathrm{eV}$. This flux mimics one from hidden-sector topological defects with $M_{X}=4 \times 10^{14} \mathrm{GeV}$ (cf. Fig. 1 (bottom)) and is also sufficient to explain the EHECR's above $E_{\mathrm{GZK}}$ via the Z-burst mechanism. Curves are without (dotted) and with relic neutrino absorption. Assumed neutrino masses are degenerate at $m_{\nu_{1}}=0.2 \mathrm{eV}$ (dashed) and $m_{\nu_{1}}=0.4 \mathrm{eV}$ (solid). The error bars indicate the statistical accuracy achievable per energy decade by the year 2013, for a flux which saturates today's observational bound from Fig. 1 (bottom).

gies $E_{p \max } \gtrsim 10^{23} \mathrm{eV}[24,25]$. Much more favorable in this respect are top-down sources, involving physics beyond the Standard Model (SM) which naturally produce extremely-high energetic particles. With either bottomup or top-down, the sources must be "hidden" [98], in order to avoid the cascade limit (cf. Fig. 1 (bottom)). Hidden sources are, by definition, sources from which neither nucleons nor photons (with energies $\gtrsim 100 \mathrm{MeV}$ ) escape.

One popular hidden top-down example is a topological defect, here taken with mass $M_{X} \sim 4 \times 10^{14} \mathrm{GeV}$, which couples to SM particles only indirectly through a non-SM sector [50]. In this case of topological-defect origin, we have $n-\alpha \simeq 0$ and $z_{\max } \gg 1$, and so Fig. 7 applies; the dip presented there is $\sim 15 \%$ for the most interesting case of quasi-degenerate neutrinos, $m_{\nu_{1}} \gtrsim 0.1 \mathrm{eV}$. In Fig. 9, we show the significant wiggle from quasidegenerate neutrinos in the otherwise power-law spectrum expected for the hidden topological-defect neutrino flux as in Fig. 1 (bottom). By design, this flux scratches the observational upper bound. Such a flux is also sufficient to explain the EHECR's via the Z-burst mechanism. The indicated error bars show the statistical significance that is expected with planned and proposed experiments by the year 2013 (cf. Fig. 1 (bottom)). This example illustrates concretely our previous claim that substantial evidence for a relic neutrino absorption dip and the existence of the $\mathrm{C} \nu \mathrm{B}$ is achievable within the foreseeable 


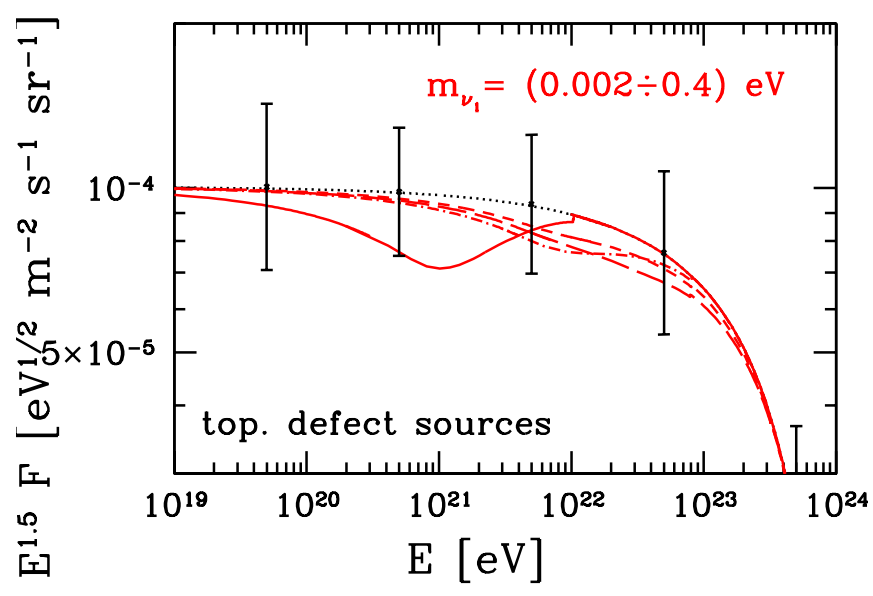

FIG. 10: As in Fig. 9, but with $E_{\max }=10^{24} \mathrm{eV}$, to mimic topological defects with $M_{X}=10^{16} \mathrm{GeV}$ (cf. Fig. 1 (bottom)). The assumed neutrino spectra are: (i) quasi-degenerate, $m_{\nu_{1}}=0.4 \mathrm{eV}$ (solid), (ii) normal hierarchical, $m_{\nu_{1}}=0.01 \mathrm{eV}$ (long-dashed) and $m_{\nu_{1}}=0.002 \mathrm{eV}$ (short-dashed), and (iii) inverted hierarchical, $m_{\nu_{1}}=0.002 \mathrm{eV}$ (dashed-dotted). Here the error bars indicate the statistical accuracy achievable per energy decade by the year 2013, for a flux which saturates today's cascade limit from Fig. 1 (bottom).

future, if the Z-burst mechanism for the EHECR's is realized in Nature.

For a hierarchical neutrino spectrum, on the other hand, with $m_{\nu_{1}} \lesssim 0.04 \mathrm{eV}$, the lowest resonant-energy is $\sim 10^{23} \mathrm{eV}$. Even if the EHEC $\nu$ flux saturates the current observational limit, the expected event numbers in the $10^{22 \div 23}$ eV energy interval are apparently too small (cf. Table I) to allow a discovery of absorption dips in the next decade: with 90 expected events in this energy range by 2013, one needs absorption depths of $\sim 53 \%$ $(\sim 32 \%)$ for a 5 -sigma discovery (3-sigma evidence). As can be gleaned from Fig. 7, such large dips are not expected.

\section{Less optimistic scenario: transparent sources}

Let us turn our attention now to the non-optimized $\mathrm{EHEC} \nu$ fluxes. From a neutrino flux that saturates the cascade limit (cf. Fig. 1 (bottom)), we may expect, in the $10^{21 \div 22} \mathrm{eV}$ energy bin, just 40 events by the year 2013 (cf. Table I). This yields a 3-sigma evidence for an absorption dip only if it has a depth of $\sim 48 \%$ [99]. Dips of this depth we have seen in $\S$ II C only for quasi-degenerate neutrinos and extreme parameter choices for the source activity. For example, for power indices $n-\alpha \gtrsim 2$, as might happen with highly-evolving bottom-up sources, one needs $z_{\max } \gtrsim 20$ in addition (cf. Figs. 4 and 8 (top)). The latter is easily achieved in topdown sources $\left(z_{\max }=\infty\right)$, however their restriction to $n-\alpha \simeq 0$ tends to decrease the depth of the dip (cf.

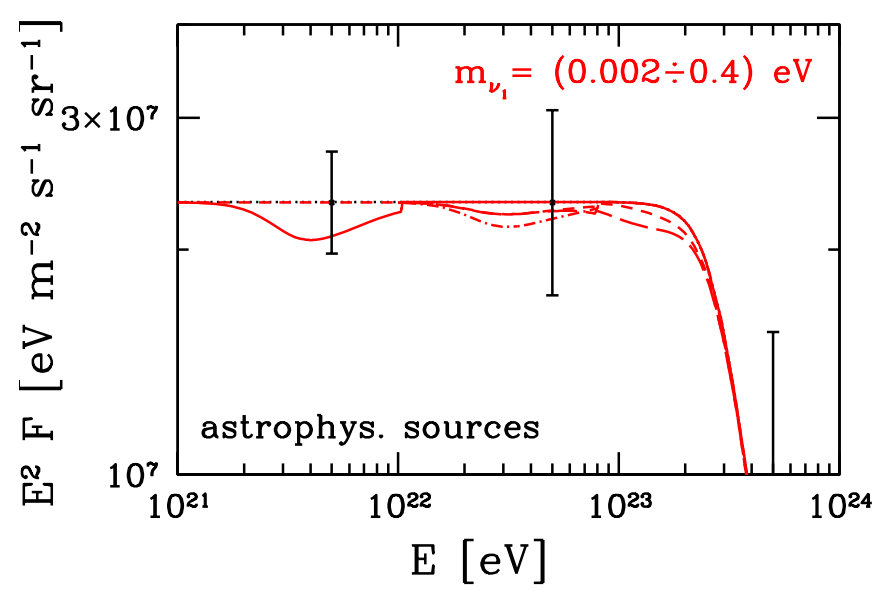

FIG. 11: As in Fig. 10, but with a generous SFR activity (25), $z_{\max }=20$, injection spectrum index $\alpha=2$, and $E_{\max }=$ $10^{24} \mathrm{eV}$, to mimic astrophysical sources with $E_{p \max }=2 \times$ $10^{16} \mathrm{GeV}$.

Figs. 4 and 7 (top)). This pessimistic outlook is however ameliorated when one realizes that an increase in statistics by a factor of 10 reduces the required absorption depth by a factor of $\sim 3$, to $\sim 15 \%$ for a 3 -sigma evidence, and to $\sim 25 \%$ for a 5 -sigma discovery. Such an increase in statistics could be achieved, for example, by undertaking more ANITA flights, by extending the EUSO flight time, or by developing the OWL or SalSA experiments. If the $\sim 13$ neutrinos in the $10^{21 \div 22} \mathrm{eV}$ interval (cf. Table I), expected from a flux which saturates the cascade limit, are measured by the pre-2008 experiments, then such extensions of the upcoming EHEC $\nu$ observatories would be warranted.

What classes of sources could deliver EHEC $\nu$ fluxes which saturate the cascade limit? It has recently been pointed out that cosmogenic neutrino fluxes from photopion production by cosmic protons on the $\mathrm{CMB}$ can reach this limit $[46,61]$. However, the limit is scratched typically just above $E_{\mathrm{GZK}}$, at around $10^{20} \mathrm{eV}$ and not higher (cf. Fig. 1 (bottom)). Other possible sources include bottom-up Zevatrons with a large $E_{p \max } \gtrsim 10^{23} \mathrm{eV}$, and topological defects with $M_{X} \gtrsim 10^{14} \mathrm{GeV}$.

As examples, we show in Figs. 10 and 11, for various neutrino mass spectra, the predicted modulations in the otherwise power-law spectra expected for a topological defect and an astrophysical neutrino flux, respectively, both saturating the cascade limit. As expected, we see that a further increase in statistics by a factor of $\sim 10 \div$ 100 , to reduce the error bars by a factor of $\sim 3 \div 10$, will be mandatory to establish absorption dips. Moreover, a quasi-degenerate neutrino mass spectrum is required. 


\section{B. Further experimental issues}

To temper optimism, it is fair to mention three further issues that mitigate an experiment's ability to cleanly observe an absorption dip. These are (i) the resolution with which an experiment can reconstruct the initial neutrino energy from the visible event energy, (ii) the flavordependent nature of energy reconstruction, and (iii) the possible confusion of a deviation of the (assumed) powerlaw spectrum due to absorption with other possible origins of deviation. We discuss each of these in turn.

Proposed EHEC $\nu$ detectors will measure shower energy from ground-based scintillator or water, or atmospheric nitrogen fluorescence, or radio signals in ice. From EHECR physics, energy reconstruction for hadroninitiated showers is known to be about $25 \%$. Neutrino observatories expect to reconstruct shower energies with about the same error. This will smear the dip somewhat, but our analysis is based upon events per decade in energy, so the $25 \%$ smearing should be tolerable.

However, there is a subtlety connected with the various showers that result from different neutrino flavors [100]. Consider first neutral current (NC) events, identical for all neutrino flavors. On average, the final state neutrino carries away $80 \%$ of the incident energy, leaving $20 \%$ in the detected jet. The NC cross-section is $\sim 45 \%$ that of the charged-current (CC) cross-section. Next consider CC interactions. The $\nu_{\mu}$ and $\nu_{\tau} \mathrm{CC}$ events produce charged leptons, generally unobservable, which carry away $80 \%$ of the energy. Thus, the $\nu_{\mu}$ and $\nu_{\tau}$ CC events leave the same $20 \%$ energy deposition in the shower as do the NC interactions. For all these events, the absorption dip in the shower events will appear lower in energy than the true dip by a factor $\sim 0.2$. This is easily corrected. However, there remains some smearing due to the event-by-event variance of the energy transfer about the mean. We do not include the effect of this variance in the present work.

In contrast to the above event classes, a $\nu_{e} \mathrm{CC}$ event will produce a hadronic jet plus an electron which creates an electromagnetic jet, and so the interaction deposits $100 \%$ of the incident energy into the combined shower.

In summary of this flavor discussion, $\mathrm{NC}$, and $\nu_{\mu}$ and $\nu_{\tau}$ CC scattering constitute about $77 \%$ of the events. The showers from these events contain about $20 \%$ of the incident neutrino energy. The other $23 \%$ of events are due to $\nu_{e} \mathrm{CC}$, with all of the incident energy observed. Thus, the observed events will look like a superposition of two fluxes with relative weights $23 \%$ and $77 \%$, the latter displaced downward in energy by a factor of five in the mean, but with fluctuations.

Finally we come to the confidence issue, whether an observed feature near the end of the EHEC $\nu$ spectrum can be claimed to be an absorption dip. For example, the spectral end-point may have structure simply due to differences among the individual contributing sources. To better ensure that a dip feature is observed, the continuation of the spectrum above the dip region should be observed. This requires more events, at higher energies still. Looking again at Table I, one sees even more reason to doubt the observation of an absorption feature, except for relatively low resonance-energies. In turn, this means that quasi-degenerate neutrinos, with mass not much below present cosmological bounds, are required if the relic neutrinos are to be detected via an absorption dip.

\section{SUMMARY AND CONCLUSIONS}

Relic neutrino absorption spectroscopy via the observation of absorption dips in the $\mathrm{EHEC} \nu$ flux may be feasible. The Z-dips, if observed, are rich in particle and astrophysical information. The position of the high-energy edge of each dip is fixed by the neutrino mass. The depth and shape are determined by the density of the $\mathrm{C} \nu \mathrm{B}$, by the usual cosmological parameters, by the activity and injection spectrum of the EHEC $\nu$ sources, and by the neutrino mass pattern. Moreover, as we have seen, the width of the dip region reflects the spectrum and evolutionary history of the neutrino source(s). Thus, were we so fortunate as to resolve a dip in some detail, the information to be mined truly belongs to interdisciplinary particle-astrophysics.

However, the statistics at the dip is entirely determined by the magnitude of the EHEC $\nu$ flux. Moreover, the energy positions of the dips - and, to some extent, their depths - critically depend on the neutrino mass spectrum.

Large event samples, $N \gg 100$, beyond $10^{21} \mathrm{eV}$ are needed to reveal Z-dips with statistical significance. To get these event numbers within the next decade or so, an $\mathrm{EHEC} \nu$ flux at least as large as the present cascade limit is required. Almost certainly, even higher fluxes must be invoked. They could be generated by hidden sources, which are opaque to nucleons and high-energy photons, thereby evading the cascade limit. Such sources are likely indicative of new physics. Moreover, the high flux requirement implies a Z-burst contribution to the EHECR events beyond $E_{\mathrm{GZK}}$ within an order of magnitude of the present AGASA rate. The Auger project should detect these large $\mathrm{EHEC} \nu$ and EHECR fluxes within a few years, and EUSO should easily measure them within the decade.

As inferred from our numerous figures, a quasidegenerate neutrino mass spectrum with $m_{\nu_{1}} \gtrsim 0.1 \mathrm{eV}$ seems to be required to produce a detectably-deep absorption dip at a possibly-accessible resonant energy. Such a spectrum is testable in several ways [9]. Neutrinoless double beta decay experiments (assuming neutrinos are Majorana) such as CUORE [74] and NEMO-3 [75], and the KATRIN tritium decay experiment [76] can be expected to show positive results already in the upcoming decade. The transfer functions relating CMB fluctuations to today's large-scale matter distributions can be expected to show positive contributions from massive neutrinos [77]. It seems sensible to say that, if pre-2008 
experiments do not see any EHEC $\nu$ flux in the $10^{21 \div 23} \mathrm{eV}$ region, then, in the context of the concordance cosmological model, absorption dips won't be observed within the next decade or two. If such is the case, then Nature will have overlooked a wonderful opportunity to produce direct evidence for the $\mathrm{C} \nu \mathrm{B}[101]$.

In summary, the presently planned neutrino detectors open up a window of opportunity for relic neutrino absorption spectroscopy. The next decade will be really exciting and decisive in this respect.

\section{APPENDIX: IMPLEMENTING NEUTRINO FLAVOR PHYSICS}

At production in the cosmic sources, let the ratios of the neutrino flavors be written as $\nu_{e}: \nu_{\mu}: \nu_{\tau}=w_{e}$ : $w_{\mu}: w_{\tau}$, with $\sum w_{\beta}=1$. A convenient description of the flavor mixture is given by the density matrix

$$
\rho(t=0)=\sum_{\beta} w_{\beta}\left|\nu_{\beta}\right\rangle\left\langle\nu_{\beta}\right|
$$

The density matrix is properly normalized to $\operatorname{Tr}(\rho(0)=$ 1 , and so describes the ensemble-averaged, single neutrino.

The relic neutrinos, with thermal energies $\sim 3 k T_{\nu} \sim$ $0.5 \mathrm{meV}$ in the today's epoch, long ago decohered into their mass-eigenstates [21]. Therefore, rewriting the density matrix in the mass-basis allows us to simply include the losses due to resonant absorption. Making use of the mixing matrix notation $\left|\nu_{\beta}\right\rangle=U_{\beta j}\left|\nu_{j}\right\rangle$, or equivalently, $U_{\beta j}=\left\langle\nu_{j} \mid \nu_{\beta}\right\rangle=\left\langle\nu_{\beta} \mid \nu_{j}\right\rangle^{*}$, the forward-propagated density matrix in the mass basis, with resonant absorption, is:

$$
\begin{aligned}
\rho(t) & =\sum_{\beta} w_{\beta} \sum_{j}\left[\mathrm{e}^{-\mathrm{i} \frac{m_{j}^{2}}{2 E} t} \mathrm{e}^{-\frac{\Gamma_{j}}{2} t} U_{\beta j}\left|\nu_{j}\right\rangle\right] \sum_{k}\left[\left\langle\nu_{k}\right| U_{\beta k}^{*} \mathrm{e}^{+\mathrm{i} \frac{m_{k}^{2}}{2 E} t} \mathrm{e}^{-\frac{\Gamma_{k}}{2} t}\right] \\
& =\sum_{\beta} w_{\beta} \sum_{j, k} U_{\beta j} U_{\beta k}^{*} \mathrm{e}^{+\mathrm{i} \Delta m_{k j}^{2} t} \mathrm{e}^{-\frac{\Gamma_{j}+\Gamma_{k}}{2} t}\left|\nu_{j}\right\rangle\left\langle\nu_{k}\right| .
\end{aligned}
$$

Here, $\Gamma_{j}=c n_{j} \sigma_{j}$ is the annihilation rate, and the factor $e^{-\frac{\Gamma}{2} t}$ correctly accounts for absorption at the amplitude level [102]. As written, this formula does not include en- ergy losses due to redshifting; however, it is straightforward to incorporate redshifting in the final expressions.

At Earth, the probability to detect flavor $\alpha$ is then,

$$
P_{\nu_{\alpha} \text { detected }}=\left\langle\nu_{\alpha}|\rho(t)| \nu_{\alpha}\right\rangle=\sum_{\beta} w_{\beta} \sum_{j, k} U_{\beta j} U_{\beta k}^{*} U_{\alpha k} U_{\alpha j}^{*} \mathrm{e}^{-\mathrm{i} \triangle m_{k j}^{2} t} \mathrm{e}^{-\frac{\Gamma_{j}+\Gamma_{k}}{2} t}
$$

This probability expression naturally divides into a sum diagonal in the mass, and a sum of interfering mass terms from which oscillations arise. After a bit of algebra, the result is:

$$
\begin{aligned}
P_{\nu_{\alpha} \text { detected }} & =\sum_{\beta} w_{\beta} \sum_{j}\left|U_{\alpha j}\right|^{2}\left|U_{\beta j}\right|^{2} \mathrm{e}^{-\Gamma_{j} t} \\
& +2 \sum_{\beta} w_{\beta} \sum_{j<k} \mathrm{e}^{-\frac{\Gamma_{j}+\Gamma_{k}}{2} t}\left[\Re\left(U_{\beta j} U_{\beta k}^{*} U_{\alpha k} U_{\alpha k}^{*}\right) \cos \left(\frac{\triangle m_{k j}^{2} t}{2 E}\right)-\Im\left(U_{\beta j} U_{\beta k}^{*} U_{\alpha k} U_{\alpha j}^{*}\right) \sin \left(\frac{\triangle m_{k j}^{2} t}{2 E}\right)\right] .
\end{aligned}
$$


Although the neutrino wave-function at extreme highenergies may remain coherent over a cosmic distance, the oscillating terms are effectively averaged away in any realistic observation. Let us look first at the coherence of the neutrino wave-function. The spread in the neutrino mass-eigenstates after travel through a distance $D$ results from the difference in the group velocities $\beta=\delta E / \delta p$ :

$$
\begin{aligned}
D_{\text {spread }} & =c t \delta \beta=\frac{D \triangle m^{2}}{2 E^{2}} \\
& =6 \times 10^{-20}\left(\frac{0.7}{h}\right)\left(\frac{D}{D_{H}}\right) \triangle m_{-3}^{2} E_{22}^{-2} \mathrm{~cm}
\end{aligned}
$$

where $\triangle m_{-3}^{2} \equiv \Delta m^{2} / 10^{-3} \mathrm{eV}^{2}, E_{22} \equiv E / 10^{22} \mathrm{eV}$, and the Hubble distance $D_{H} \equiv c H_{0}^{-1}=4.2(0.7 / h)$ Gpc characterizes the typical cosmic distance. Decoherence of the wave packet results when $D_{\text {spread }}$ exceeds the natural length of the wavepacket, call it $c \tau_{\Psi}$, the latter being determined by conditions at the source [103]. The natural length may be $c$ times the production time [80] (e.g. $c \tau_{\pi^{ \pm}} \sim 3 \mathrm{~m}$ ), the mfp between interactions in a dense source [80], or the spatial uncertainty in the location of the production point in the source [81]. The decoherence length is obtained by setting $D_{\text {spread }}$ in Eq. (A.5) equal to $c \tau_{\Psi}$, and solving for $D$. The result, as a fraction of the Hubble size, is

$$
\frac{D_{\text {decohere }}}{D_{H}}=0.5\left(\frac{h}{0.7}\right)\left(\frac{\tau_{\Psi}}{3 \mathrm{~m}}\right) \frac{E_{22}^{2}}{\triangle m_{-3}^{2}} \times 10^{20} .
$$

Clearly, decoherence does not occur in our Universe for a $10^{22} \mathrm{eV}$ neutrino.

Now we turn to the averaging effects of measurement. To observe the oscillating terms requires measurement of the phase $\phi=\triangle m^{2} t / 2 E$ to better than $2 \pi$, or equivalently, knowledge of $\delta(D / E)$ to better than $4 \pi / \Delta m^{2}$. This in turn requires knowledge of $\delta D$ to better than $\lambda_{\text {osc }} \equiv 4 \pi E / \triangle m^{2}$, and knowledge of $\delta E$ to better than $E\left(\lambda_{\text {osc }} / D\right)$. With the oscillation length being so short $\sim E_{22} / \triangle m_{-3}^{2} \mathrm{kpc}$ compared to cosmic scales, there is no hope to observe the oscillating term.

From here on, we may use for the detection probability just the averaged value of Eq. (A.4), i.e. just the first term

$$
P_{\nu_{\alpha} \text { detected }}=\sum_{j}\left|U_{\alpha j}\right|^{2} e^{-\Gamma_{j} t} \sum_{\beta} w_{\beta}\left|U_{\beta j}\right|^{2} .
$$

Finally, taking into account energy losses due to redshift, Eq. (A.7) is easily generalized to Eq. (9) in the main text for the differential propagation function of neutrinos, and to Eq. (17) for the neutrino flux to be observed at Earth.

As simple as Eq. (A.7) is, further simplifications are possible. For example, if $w_{e}: w_{\mu}: w_{\tau}=1: 1: 1$, as might arise from "democratic" neutrino emission from a topological defect, then by unitarity of the mixing matrix, $\sum_{\beta} w_{\beta}\left|U_{\beta j}\right|^{2}=1 / 3$ independently of $j$. It is also known [33] that the same result obtains for the case $w_{e}: w_{\mu}: w_{\tau}=1: 2: 0$ as results from charged pion decay to neutrinos, in the limit of $\nu_{\mu} \leftrightarrow \nu_{\tau}$ interchangesymmetry. The latter is exact if the atmospheric mixing angle $\theta_{32}$ is maximal $\left(45^{\circ}\right)$ and $\Re\left(U_{e 3}\right)=0$. Oscillation data [5] show that these two conditions are satisfied (or nearly so). Since any linear combination of the two sets $\left\{w_{\beta}\right\}$ just discussed must also give the same conclusion, we arrive at a mini-Theorem:

whenever $w_{e}=1 / 3$ and $\nu_{\mu} \leftrightarrow \nu_{\tau}$ interchange-symmetry is (nearly) valid,

then $\sum_{\beta} w_{\beta}\left|U_{\beta j}\right|^{2}=1 / 3$ independently of $j$.

Because $w_{e}=1 / 3$ includes two popular neutrinoproduction cases, charged pion decay and democratic emission, we will assume that $w_{e}=1 / 3$ holds and work with the the very compact result

$$
P_{\nu_{\alpha} \text { detected }}=\frac{1}{3} \sum_{j}\left|U_{\alpha j}\right|^{2} e^{-\Gamma_{j} t} .
$$

This expression leads immediately to the main text's Eq. (19) for the neutrino flux at Earth, after again taking into account the energy loss due to redshifting.

Cosmic-neutrino detectors are unlikely to be capable of neutrino flavor identification (see Ref. [51] for a discussion). Accordingly, it is tempting to also sum over $\alpha$, the flavor arriving at Earth, to arrive at the especially simple result

$$
P_{\nu_{\text {any }}}=\sum_{\alpha} P_{\nu_{\alpha} \text { detected }}=\frac{1}{3} \sum_{j} e^{-\Gamma_{j} t},
$$

leading to Eq. (21). Caution is warranted, however, as the interactions of the different flavors deposit different mean energies into the detector. For example, the short mean-free-path (mfp) of a high-energy electron, and the long mfp's of high-energy muons and taus, imply that in charged-current interactions, $\nu_{\mu}$ 's and $\nu_{\tau}$ 's will leave much less visible energy in the detector than will $\nu_{e}$ 's. This issue is discussed further in the main text in $\S$ III.

\section{ACKNOWLEDGMENTS}

We thank Luis Anchordoqui, Sidney Bludman, Sergio Bottai, Zoltan Fodor, Peter Gorham, Steen Hannestad, Michael Kachelriess, Sandor Katz, Chris Quigg, Christian Spiering, and Yvonne Wong for useful discussions and important information. Help in programming issues from Hui Fang is also kindly acknowledged. TJW thanks the CfCP at the U. Chicago, KIPAC at Stanford/SLAC, US DoE grant DE-FG05-85ER40226, NASA-ATP grant 02-0000-0151, and Vanderbilt University's Discovery Grant program for sabbatical support during preparation of this paper. 
[1] T. J. Weiler, Phys. Rev. Lett. 49, 234 (1982); Neutrino Mass and Gauge Structure of Weak Interactions, Telemark, USA, 1982, pp. 60-75.

[2] T. J. Weiler, Astrophys. J. 285, 495 (1984).

[3] E. Roulet, Phys. Rev. D 47, 5247 (1993).

[4] S. Yoshida, H. Dai, C. C. Jui and P. Sommers, Astrophys. J. 479, 547 (1997).

[5] K. Hagiwara et al. [Particle Data Group Collaboration], Phys. Rev. D 66, 010001 (2002).

[6] J. Bernstein, M. Ruderman and G. Feinberg, Phys. Rev. 132, 1227 (1963);

B. P. Konstantinov and G. E. Kocharov, J. Exp. Theor. Phys. 19, 992 (1964);

R. Cowsik, Y. Pal and S. N. Tandon, Phys. Lett. 13, 265 (1964);

T. Hara and H. Sato, Prog. Theor. Phys. 64, 1089 (1980);

Prog. Theor. Phys. 65, 477 (1981).

[7] R. Wigmans, Astropart. Phys. 19, 379 (2003);

E. A. Paschos and O. Lalakulich, hep-ph/0206273;

S. Davidson, S. Forte, P. Gambino, N. Rius and A. Strumia, JHEP 0202, 037 (2002).

[8] A. Ringwald, in: Proc. Workshop on Strong and Electroweak Matter 2002, Heidelberg, Germany, 2-5 Oct 2002, ed. M. G. Schmidt, World Scientific, pp. 497-503, hep-ph/0301157.

[9] H. Päs and T. J. Weiler, Phys. Rev. D 63, 113015 (2001);

S. M. Bilenky, C. Giunti, J. A. Grifols and E. Masso, Phys. Rept. 379, 69 (2003).

[10] D. N. Spergel et al., Astrophys. J. Suppl. 148, 175 (2003).

[11] M. Tegmark et al. [SDSS Collaboration], astro$\mathrm{ph} / 0310723$.

[12] S. Hannestad, JCAP 0305, 004 (2003);

O. Elgaroy and O. Lahav, JCAP 0304, 004 (2003);

V. Barger, D. Marfatia and A. Tregre, hep-ph/0312065.

[13] S. W. Allen, R. W. Schmidt and S. L. Bridle, astroph/0306386.

[14] V. D. Barger, T. J. Weiler and K. Whisnant, Phys. Lett. B 442, 255 (1998).

[15] C. Weinheimer et al., Phys. Lett. B 460, 219 (1999); V. M. Lobashev et al., Phys. Lett. B 460, 227 (1999); C. Weinheimer, Nucl. Phys. Proc. Suppl. 118, 279 (2003).

[16] H. V. Klapdor-Kleingrothaus et al., Eur. Phys. J. A 12, 147 (2001);

C. E. Aalseth et al. [IGEX Collaboration], Phys. Rev. D 65, 092007 (2002).

[17] H. V. Klapdor-Kleingrothaus, A. Dietz, H. L. Harney and I. V. Krivosheina, Mod. Phys. Lett. A 16, 2409 (2001);

H. V. Klapdor-Kleingrothaus, A. Dietz, I. V. Krivosheina, C. Doerr and C. Tomei, Phys. Lett. B 578, 54 (2004).

[18] F. Feruglio, A. Strumia and F. Vissani, Nucl. Phys. B 637, 345 (2002) [Addendum-ibid. B 659, 359 (2003)];

C. E. Aalseth et al., Mod. Phys. Lett. A 17, 1475 (2002).

[19] K. Greisen, Phys. Rev. Lett. 16, 748 (1966); G. T. Zatsepin and V. A. Kuzmin, JETP Lett. 4, 78 (1966) [Pisma Zh. Eksp. Teor. Fiz. 4, 114 (1966)].
[20] T. J. Weiler, Astropart. Phys. 11, 303 (1999).

[21] T. J. Weiler, in: Proc. Beyond the Desert 99', Ringberg Castle, Tegernsee, Germany, June 6-12, 1999, ed. H. Klapdor-Kleingrothaus, hep-ph/9910316.

[22] D. Fargion, B. Mele and A. Salis, Astrophys. J. 517, 725 (1999).

[23] S. Yoshida, G. Sigl and S. J. Lee, Phys. Rev. Lett. 81, 5505 (1998).

[24] Z. Fodor, S. D. Katz and A. Ringwald, Phys. Rev. Lett. 88, 171101 (2002); JHEP 0206, 046 (2002).

[25] O. E. Kalashev, V. A. Kuzmin, D. V. Semikoz and G. Sigl, Phys. Rev. D 65, 103003 (2002);

D. S. Gorbunov, P. G. Tinyakov and S. V. Troitsky, Astropart. Phys. 18, 463 (2003);

D. V. Semikoz and G. Sigl, hep-ph/0309328.

[26] Pierre Auger Observatory, http://www.auger.org/

[27] IceCube, http://icecube.wisc.edu/

[28] ANtarctic Impulse Transient Array, http://www.ps.uci.edu/ anita/

[29] Extreme Universe Space Observatory, http://www.euso-mission.org/

[30] Orbiting Wide-angle Light-collectors, http://owl.gsfc.nasa.gov/

[31] Saltdome Shower Array, P. Gorham et al., Nucl. Instrum. Meth. A 490, 476 (2002).

[32] C. Spiering, J. Phys. G 29, 843 (2003); private communication.

[33] J. G. Learned and S. Pakvasa, Astropart. Phys. 3, 267 (1995);

L. Bento, P. Keranen and J. Maalampi, Phys. Lett. B 476, 205 (2000);

H. Athar, M. Jezabek and O. Yasuda, Phys. Rev. D 62, 103007 (2000).

[34] Radio Ice Cerenkov Experiment,

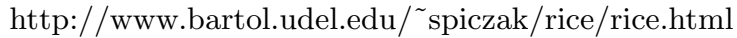

[35] Goldstone Lunar Ultra-high energy neutrino Experiment, http://www.physics.ucla.edu/ moonemp/public/

[36] Fast On-orbit Recording of Transient Events satellite, A. R. Jacobson, S. O. Knox, R. Franz and D. C. Enemark, Radio Sci. 34, 337 (1999).

[37] I. Kravchenko, astro-ph/0306408.

[38] P. W. Gorham, C. L. Hebert, K. M. Liewer, C. J. Naudet, D. Saltzberg and D. Williams, astro$\mathrm{ph} / 0310232$.

[39] N. G. Lehtinen, P. W. Gorham, A. R. Jacobson and R. A. Roussel-Dupre, astro-ph/0309656.

[40] R. M. Baltrusaitis et al., Phys. Rev. D 31, 2192 (1985); S. Yoshida et al. [AGASA Collaboration], in: Proc. 27th International Cosmic Ray Conference, Hamburg, Germany, 2001, Vol. 3, pp. 1142-1145;

L. A. Anchordoqui, J. L. Feng, H. Goldberg and A. D. Shapere, Phys. Rev. D 66, 103002 (2002).

[41] X. Bertou, P. Billoir, O. Deligny, C. Lachaud and A. Letessier-Selvon, Astropart. Phys. 17, 183 (2002).

[42] P. Gorham et al., [ANITA collaboration], Proposal SMEX03-0004-0019, April 19, 2003.

[43] S. Bottai and S. Giurgola [EUSO Collaboration], in: Proc. 28th International Cosmic Ray Conference, Tsukuba, Japan, 2003, pp. 1113-1116; 
S. Bottai [EUSO Collaboration], to appear in: Proc. Incontro Nazionale di Astrofisica delle Alte Energie, Roma, May 15-16, 2003.

[44] P. Gorham, private communication.

[45] S. Yoshida and M. Teshima, Prog. Theor. Phys. 89, 833 (1993);

J. N. Bahcall and E. Waxman, Astrophys. J. 542, 543 (2000);

Z. Fodor and S. D. Katz, Phys. Rev. D 63, 023002 (2001);

Z. Fodor, S. D. Katz, A. Ringwald and H. Tu, Phys. Lett. B 561, 191 (2003);

Z. Fodor, S. D. Katz and A. Ringwald, in preparation.

[46] Z. Fodor, S. D. Katz, A. Ringwald and H. Tu, JCAP 0311, 015 (2003).

[47] A. Blanchard, M. Douspis, M. Rowan-Robinson and S. Sarkar, Astron. Astrophys. 412, 35 (2003).

[48] A. D. Dolgov, S. H. Hansen, S. Pastor, S. T. Petcov, G. G. Raffelt and D. V. Semikoz, Nucl. Phys. B 632, 363 (2002);

Y. Y. Wong, Phys. Rev. D 66, 025015 (2002);

K. N. Abazajian, J. F. Beacom and N. F. Bell, Phys. Rev. D 66, 013008 (2002);

V. Barger, J. P. Kneller, P. Langacker, D. Marfatia and G. Steigman, Phys. Lett. B 569, 123 (2003).

[49] S. Singh and C. P. Ma, Phys. Rev. D 67, 023506 (2003).

[50] V. S. Berezinsky and A. Vilenkin, Phys. Rev. D 62, 083512 (2000);

V. Berezinsky, M. Narayan and F. Vissani, Nucl. Phys. B 658, 254 (2003).

[51] J. F. Beacom, N. F. Bell, D. Hooper, S. Pakvasa and T. J. Weiler, Phys. Rev. D 68, 093005 (2003).

[52] J. F. Beacom and N. F. Bell, Phys. Rev. D 65, 113009 (2002).

[53] C. Giunti and M. Laveder, to be published in: Progress in Quantum Physics Research, V. Krasnoholovets (ed.), Nova Science Publishers, Inc., hep-ph/0310238.

[54] S. D. Wick, C. D. Dermer and A. Atoyan, astro$\mathrm{ph} / 0310667$.

[55] P. Madau, L. Pozzetti and M. Dickinson, Astrophys. J. 498, 106 (1998).

[56] A. W. Blain and P. Natarajan, Mon. Not. R. Astron. Soc. 309, 715 (1999).

[57] P. Bhattacharjee, C. T. Hill and D. N. Schramm, Phys. Rev. Lett. 69, 567 (1992).

[58] P. Chen, T. Tajima and Y. Takahashi, Phys. Rev. Lett. 89, 161101 (2002).

[59] S. A. Colgate, talk at the 2002 Aspen Winter Conference Ultrahigh Energy Particles from Space, Jan. 27-Feb. 2; based on dynamics in

H. Li, S. A. Colgate, M. Kusunose and R. V. E. Lovelace, in: Proc. High Energy Processes in Accreting Black Holes, eds. J .Poutanen and R. Svensson, ASP Conf. Series, astro-ph/9812418;

P. P. Kronberg, Q. W. Dufton, H. Li and S. A. Colgate, Astrophys. J., accepted, astro-ph/0106281.

[60] K. Mannheim, Astropart. Phys. 3, 295 (1995).

[61] O. E. Kalashev, V. A. Kuzmin, D. V. Semikoz and G. Sigl, Phys. Rev. D 66, 063004 (2002);

D. V. Semikoz and G. Sigl, hep-ph/0309328.

[62] V. Berezinsky, hep-ph/0303091;

R. Aloisio, V. Berezinsky and M. Kachelriess, hepph/0307279;

R. Aloisio, V. Berezinsky and P. Blasi, in preparation.
[63] V. S. Berezinsky and A. Yu. Smirnov, Astrophysics and Space Science 32, 461 (1975).

[64] K. Mannheim, R. J. Protheroe and J. P. Rachen, Phys. Rev. D 63, 023003 (2001).

[65] V. Berezinsky, M. Kachelriess and S. Ostapchenko, Phys. Rev. Lett. 89, 171802 (2002).

[66] P. Sreekumar et al., Astrophys. J. 494 (1998) 523.

[67] A. W. Strong, I. V. Moskalenko and O. Reimer, astro$\mathrm{ph} / 0306345$;

U. Keshet, E. Waxman and A. Loeb, astro-ph/0306442.

[68] Gamma-ray Large Area Space Telescope, http://glast.gsfc.nasa.gov/

[69] Constellation-X, http://constellation.gsfc.nasa.gov/

[70] V. S. Berezinsky, S. V. Bulanov, V. A. Dogiel, V. L. Ginzburg and V. S. Ptuskin, Astrophysics of Cosmic Rays, North-Holland, 1990.

[71] V. S. Berezinsky and V. I. Dokuchaev, Astropart. Phys. 15, 87 (2001).

[72] N. Gehrels, Astrophys. J. 303, 336 (1986); building on

V. H. Regener, Phys. Rev. 84, 161 (1951); see also the statistics section of Ref. [5].

[73] R. Gandhi, C. Quigg, M. H. Reno and I. Sarcevic, Phys. Rev. D 58, 093009 (1998); Astropart. Phys. 5, 81 (1996).

[74] Cryogenic Underground Observatory for Rare Events, http://crio.mib.infn.it/wig/Cuorepage/index.html

[75] Neutrino Ettore Majorana Observatory, http://nemo.web.lal.in2p3.fr/

[76] KArlsruhe TRItium Neutrino experiment, http://www-ik1.fzk.de/tritium/

[77] W. Hu, D. J. Eisenstein and M. Tegmark, Phys. Rev. Lett. 80, 5255 (1998).

[78] For a recent overview, see S. Kazantzidis, L. Mayer, C. Mastropietro, J. Diemand, J. Stadel and B. Moore, astro-ph/0312194.

[79] P. Palunas, H. I. Teplitz, P. J. Francis, G. M. Williger and B. E. Woodgate [Gemini Deep Deep Survey collaboration], Astrophys. J., to appear, astro-ph/0311279; K. Glazebrook et al. [Gemini Deep Deep Survey collaboration], astro-ph/0401037.

[80] S. Nussinov, Phys. Lett. B 63, 201 (1976).

[81] B. Kayser, Phys. Rev. D 24, 110 (1981).

[82] For earlier and related suggestions, see Ref. [6] and Ref. [7], respectively. For a recent review, also covering non-cosmic-ray opportunities to detect the relic neutrinos, see Ref. [8].

[83] In fact, the depths of the absorption dips are inversely proportional to the expansion rate, leading, for fixed $H_{0}$, to a significant increase in the depth by a factor of $\Omega_{M}^{-1 / 2} \sim 1.8$ at large redshift, when compared to the old cold dark matter (CDM) estimate based on $\Omega_{M}=$ $1[1,2]$ (see $\S$ II A for details). This is equivalent to an improvement in statistics by a factor of $\Omega_{M}^{-1} \sim 3$.

[84] We will label neutrino masses in the order $m_{\nu_{1}} \leq$ $m_{\nu_{2}} \leq m_{\nu_{3}}$, regardless of the type of neutrino mass spectrum ("normal" or "inverted"). In this convention, $\triangle m_{\text {atm }}^{2} \simeq \triangle m_{32}^{2}, \Delta m_{\odot}^{2} \simeq \triangle m_{21}^{2}$ in a "normal" scheme, and $\triangle m_{\text {atm }}^{2} \simeq \triangle m_{21}^{2}, \triangle m_{\odot}^{2} \simeq \triangle m_{32}^{2}$ in an "inverted" one, respectively. (We are using the obvious notation $\triangle m_{i j}^{2} \equiv m_{\nu_{i}}^{2}-m_{\nu_{j}}^{2}$.) Another convention is often used in the literature: $m_{\nu_{1}} \leq m_{\nu_{2}} \leq m_{\nu_{3}}$ in "normal" schemes, 
and $m_{\nu_{3}} \leq m_{\nu_{1}} \leq m_{\nu_{2}}$ in "inverted" schemes; this has the feature that, in either scheme, $\triangle m_{\odot}^{2} \simeq \triangle m_{21}^{2} \geq 0$ and $\triangle m_{\text {atm }}^{2} \simeq\left|\triangle m_{32}^{2}\right|$.

[85] Seemingly minor assumptions can make a crucial difference for neutrino inferences from cosmic structure evolution, as discussed in detail in Refs. [11, 12]. In this sense, the recent claim that a combination of WMAP, 2dFGRS, and X-ray galaxy cluster data prefer a nonzero neutrino mass of $\sum_{i} m_{\nu_{i}}=0.56_{-0.26}^{+0.30} \mathrm{eV}[13]$ have to be viewed with some caution.

[86] The neutrino mass parameter measured in tritium beta decay is $m_{\beta} \equiv\left(\sum_{i}\left|U_{e i}\right|^{2} m_{\nu_{i}}^{2}\right)^{\frac{1}{2}}$. Therefore, the heaviest neutrino mass is bounded by $m_{\nu_{3}}<$ $\sqrt{m_{\beta}^{2}+\triangle m_{\text {atm }}^{2}}[14]$.

[87] This bound applies only for Majorana neutrinos. For a quasi-degenerate neutrino spectrum $\left(m_{\nu_{1}} \gg \Delta m_{\text {atm }}^{2}\right)$, it arises from $m_{\nu_{3}} \leq 2\left\langle m_{\nu}\right\rangle$, where $\left\langle m_{\nu}\right\rangle$ is the effective mass $\left|\sum_{i} U_{e i}^{\overline{2}} m_{\nu_{i}}\right|$, measured in neutrinoless double beta decay, $U$ being the leptonic mixing matrix. The quoted ranges in the upper bound on $\left\langle m_{\nu}\right\rangle$ take into account the spread due to different calculations of the relevant nuclear matrix elements. A recently reported evidence for neutrinoless double beta decay [17] and correspondingly deduced parameter range $\left\langle m_{\nu}\right\rangle=0.39_{-0.28}^{+0.17} \mathrm{eV}(95 \% \mathrm{CL})$ have been challenged by Ref. [18]. Nevertheless, in our conclusions we will note that a quasi-degenerate neutrino mass above $\sim 0.1 \mathrm{eV}$ is required to produce a measurable absorption dip at an accessible resonant energy.

[88] The convenience of using $c t$ as the measure of distance, with $t$ being the look-back time, is that it is easily translated into redshift. An alternate, but equivalent, derivation of the relation between source luminosity and differential flux at Earth, using not $t$ but rather the comoving distance, is given in Ref. [2]. Note that, in this original work, the source luminosity is defined per physical volume, so there is an additional redshift dependence there, traceable to $\mathcal{L}($ per comoving volume $)=(1+z)^{-3} \mathcal{L}($ per physical volume).

[89] See, however, Ref. [47] for a viable alternative to the concordance model. This alternative, which was obtained from the relaxation of the hypothesis that the primordial fluctuation spectrum, as measured in the CMB, can be described by a single power-law, has $\Omega_{\Lambda}=0$, $\Omega_{M}=0.88, \Omega_{\nu}=0.12$, and $h=0.46$ as best-fit values. Interestingly, the amount of neutrino hot dark matter $\left(\Omega_{\nu}=0.12\right)$ needed in this model points to a quasidegenerate neutrino mass spectrum with $m_{\nu_{i}} \approx 0.8 \mathrm{eV}$.

[90] Both approximations, (i) and (ii), are very well satisfied in the energy regions of the absorption dips, i.e. the energy decade below the resonance energy, on which our analysis mainly concentrates. At energies well above the Z-resonant values, t-channel $\mathrm{W}$ and $\mathrm{Z}$ exchange becomes a dominant energy loss mechanism. These t-channel reactions are the focus of Ref. [22].

[91] Expression (12) for the relic neutrino density should be considered as a rather firm prediction. Possible uniform density enhancements due to lepton asymmetries are negligible in view of the recent, very stringent bounds on the neutrino degeneracies [48]. Moreover, significant (>10) local density enhancements due to gravitational clustering are, for the mass range (4), only expected in the innermost regions $(<100 \mathrm{kpc})$ of very massive $\left(>10^{14} M_{\odot}\right)$ - and hence rare - clusters [49].

[92] If the neutrinos are Dirac particles rather than Majorana particles, then their transition from relativistic, single-helicity particles to non-relativistic unpolarized particles populates the sterile spin-states. If such is the case, then the depolarization of the Dirac states halves the unpolarized cross-section, which in turn halves the annihilation rates compared to the rates for Majorana neutrinos $[2,21]$. We present results only for the Majorana case, since the theoretical models for neutrinomass generation favor Majorana neutrinos (two light spin-states per flavor).

[93] This is the case for almost all proposed topological defects such as ordinary strings, monopolonium, and necklaces. For superconducting cosmic strings it can be as large as $n=2$ or bigger.

[94] Any realistic injection spectrum $J_{\nu_{\beta}}$ falls off rapidly with very large energy. Consequently, the contribution of large $z$ is heavily suppressed in the relevant $z$ integration (21), because the integrand is proportional to $J_{\nu_{\beta}}(E(1+z))$. Thus, the dependence on $z_{\max }$ is weak for very large $z_{\max }$.

[95] The contribution from small $z$ in Eq. (21) is negligible, for any reasonable activity. We note that a "cosmological" distance of $50 \mathrm{Mpc}$ corresponds to the small value $z=0.012$, for our default cosmological parameters.

[96] For the case of small, i.e. non-degenerate or "hierarchical", neutrino masses (cf. Fig. 3), the respective resonance energies given in Eq. (1) may possibly spread over three orders of magnitude. In this case, it might be appropriate to consider also broken power-law injection spectra. We will not pursue this because, as will be shown below, a significant detection of absorption dips in the foreseeable future seems to be possible only if neutrino masses are quasi-degenerate.

[97] The cascade limit from Ref. [64] exploits the measurement of the diffuse $\gamma$ ray background from $30 \mathrm{MeV}$ to $100 \mathrm{GeV}$ by EGRET [66]. A lower extragalactic contribution to the $\gamma$ ray background than that inferred in Ref. [66], by roughly a factor of two, has been proposed recently [67]. The cascade limit may therefore be stronger by a corresponding factor. Also, in the next few years the GLAST experiment [68], and eventually its successors, e.g. Constellation-X [69], may resolve some of the diffuse flux, thereby lowering the cascade limit even further.

[98] Possible astrophysical hidden sources have been discussed in the textbook [70] and in Ref. [71].

[99] If the number of events are small, one should apply Poisson statistics, as given in Ref. [72].

[100] We take our numbers from the extrapolated crosssections in [73].

[101] Even if Z-dips cannot be measured, it may still be possible to infer the $\mathrm{C} \nu \mathrm{B}$ from Z-burst data. The statistics of "emission" spectroscopy are not as formidable as those of absorption spectroscopy. In emission spectroscopy well above $E_{\mathrm{GZK}}$, each event is background free, and therefore statistically significant.

Finally, let us comment on a possible loophole to greater event rates than those expressed so far. We have worked in the context of the concordance model of cosmology. There are some chinks in the armor. It has been known for some time that simulations with concordance parameters over-produce small-scale objects (dwarf galax- 
ies and satellites) compared to observation [78]. Morerecent observational evidence suggests that the largest scales may also conflict with the concordance model. There is some evidence that elliptical galaxies, galactic clusters, and even vast filaments and walls delimiting huge voids may have formed very early in the universe $(z \geq 2)$ [79]. Such precocious structure suggests topdown hierarchical development rather than bottom-up as predicted by the concordance model. The parameters of the concordance cosmology may give way to something new. All of this encourages an open mind. If large structures did form early, then more neutrino sources and strong source evolution may be the reality. With precocious clustering of matter, precocious clustering of neutrinos also becomes possible. Neutrino clustering in our Galactic Supercluster would enhance "local" absorption, and thereby create narrower and deeper Z-dips (it would also greatly enhance the local Z-burst rate).

[102] The possible extermination of Schrödinger's cat is described in the same way.

[103] There is also a quantum mechanical spreading of the wavepacket, governed by the phase velocity $E / p$. This spreading is smaller than the eigenstate separation by the tiny factor $\delta p_{\Psi} / E \sim\left(c \tau_{\Psi} E\right)^{-1}$ [81], and therefore negligible. 